\title{
Phase Noise and Wideband Transmission in Massive MIMO
}

Antonios Pitarokoilis

\section{¿ommunication Sิ̂YSTEMS}

Division of Communication Systems

Department of Electrical Engineering (ISY)

Linköping University, SE-581 83 Linköping, Sweden www.commsys.isy.liu.se

Linköping 2016 
Phase Noise and Wideband Transmission in Massive MIMO

(C) 2016 Antonios Pitarokoilis, unless otherwise noted.

ISBN 978-91-7685-791-5

ISSN 0345-7524

Printed in Sweden by LiU-Tryck, Linköping 2016 
To my parents and Kallia 



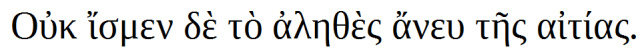

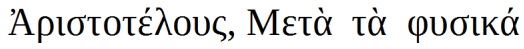

We do not know a truth without its cause.

Aristotle, Metaphysics 



\section{Abstract}

In the last decades the world has experienced a massive growth in the demand for wireless services. The recent popularity of hand-held devices with data exchange capabilities over wireless networks, such as smartphones and tablets, increased the wireless data traffic even further. This trend is not expected to cease in the foreseeable future. In fact, it is expected to accelerate as everyday apparatus unrelated with data communications, such as vehicles or household devices, are foreseen to be equipped with wireless communication capabilities.

Further, the next generation wireless networks should be designed such that they have increased spectral and energy efficiency, provide uniformly good service to all of the accommodated users and handle many more devices simultaneously. Massive multiple-input multiple-output (Massive MIMO) systems, also termed as large-scale MIMO, very large MIMO or full-dimension MIMO, have recently been proposed as a candidate technology for next generation wireless networks. In Massive MIMO, base stations (BSs) with a large number of antenna elements serve simultaneously only a few tens of single antenna, non-cooperative users. As the number of BS antennas grow large, the normalized channel vectors to the users become pairwise asymptotically orthogonal and, therefore, simple linear processing techniques are optimal. This is substantially different from the current design of contemporary cellular systems, where BSs are equipped with a few antennas and the optimal processing is complex. Consequently, the need for redesign of the communication protocols is apparent.

The deployment of Massive MIMO requires the use of many inexpensive and, potentially, off-the-shelf hardware components. Such components are likely to be of low quality and to introduce distortions to the information signal. Hence, Massive MIMO must be robust against the distortions introduced by the hardware impairments. Among the most important hardware impairments is phase noise, which is introduced by local oscillators (LOs) 
at the BS and the user terminals. Phase noise is a phenomenon of particular importance since it acts multiplicatively on the desired signal and rotates it by some random and unknown argument. Further, the promised gains of Massive MIMO can be reaped by coherent combination of estimated channel impulse responses at the BS antennas. Phase noise degrades the quality of the estimated channel impulse responses and impedes the coherent combination of the received waveforms.

In this dissertation, wideband transmission schemes and the effect of phase noise on Massive MIMO are studied. First, the use of a low-complexity single-carrier precoding scheme for the broadcast channel is investigated when the number of BS antennas is much larger than the number of served users. A rigorous, closed-form lower bound on the achievable sum-rate is derived and a scaling law on the potential radiated energy savings is stated. Further, the performance of the proposed scheme is compared with a sumcapacity upper bound and with a bound on the performance of the contemporary multi-carrier orthogonal frequency division multiplexing (OFDM) transmission.

Second, the effect of phase noise on the achievable rate performance of a wideband Massive MIMO uplink with time-reversal maximum ratio combining (TR-MRC) receive processing is investigated. A rigorous lower bound on the achievable sum-rate is derived and a scaling law on the radiated energy efficiency is established. Two distinct LO configurations at the BS, i.e., the common LO (synchronous) operation and the independent LO (non-synchronous) operation, are analyzed and compared. It is concluded that the non-synchronous operation is preferable due to an averaging of the independent phase noise sources. Further, a progressive degradation of the achievable rate due to phase noise is observed. A similar study is extended to a flat fading uplink with zero-forcing $(\mathrm{ZF})$ receiver at the BS.

The fundamental limits of data detection in a phase-noise-impaired uplink are also studied, when the channel impulse responses are estimated via uplink training. The corresponding maximum likelihood (ML) detector is provided for the synchronous and non-synchronous operations and for a general parameterization of the phase noise statistics. The symbol error rate (SER) performance at the high signal-to-noise ratio (SNR) of the detectors is studied. Finally, rigorous lower bounds on the achievable rate of a Massive MIMO-OFDM uplink are derived and scaling laws on the radiated energy efficiency are stated. 


\section{Populärvetenskaplig sammanfattning}

Det har skett en massiv tillväxt i efterfrågan på trådlösa tjänster de senaste årtiondena. Dessutom blir handhållna enheter med förmåga att kommunicera över trådlösa nätverk, som mobiltelefoner och surfplattor, allt vanligare vilket gör den trdlösa trafiken ökar ytterligare. Denna trend väntas inte upphöra inom överskådlig framtid. I själva verket förväntas den accelerera i takt med att vardagliga apparater tidigare orelaterade till kommunikation, såsom fordon eller hushållsapparater, planeras vara utrustade med trådlösa kommunikationsmöjligheter. Stora leverantörer inom den trådlösa kommunikationsindustrin förutspår en tiofaldig ökning av datatrafiken fram till år 2019 jämfört med 2014 och en tusenfaldig ökning är bara en tidsfråga.

Vidare bör nästa generation trådlösa nätverk utformas så att de har ökad effektivitet i förhållande till den tillhandahållna datahastigheten och energikonsumtionen, jämn kvalitet på tjänster till användarna samt hantera många fler enheter samtidigt. Massiv MIMO ("multiple-input multipleoutput"), även kallad hyper MIMO och storskalig MIMO, har nyligen föreslagits som en kandiderande teknik för nästa generations trådlösa nätverk. I massiv MIMO är basstationen utrustad med ett stort antal antal antenner och betjänar ett tiotal icke samarbetande,användare samtidigt. Med ökande antal antenner vid basstationen minskar störningar mellan kanaler till olika användare och enkla signalbehandlingstekniker har mycket bra prestanda.

Utbyggnaden av massiv MIMO kräver användning av många billiga och potentiellt sett icke specialgjorda komponenter. Sådana komponenter kommer sannolikt att vara av låg kvalitet och införa förvrängningar av informationssignalen. Därför måste Massiva MIMO vara robust mot dessa förvrängningar som kommer från användning av icke-ideal hårdvara. 
En av de viktigaste funktionsnedsättningarna från icke-ideal hårdvara är fasbrus. Denna funktionsnedsättning introduceras av komponenter som kallas lokala oscillatorer som finns i basstationen såväl som i användarterminalerna. Fasbrus är ett fenomen av särskild betydelse, eftersom den slumpvis roterar den önskade signalen och kan inte åtgärdas genom att öka effekten för den önskade signalen. Vidare kan de utlovade vinsterna av massiv MIMO skördas genom lämplig kombination av de mottagna signalerna vid basstationen. Emellertid förvränger rotationen från fasbruset denna kombinering.

I denna avhandling studerar vi bredbandsöverföringssystem och effekten av fasbruset på Massive MIMO. I bredbandsöverföring skiljer sig svaret från kanalen från en signal för varje frekvenskomponent. Detta resulterar $i$ en förvrängning av den mottagna signalen och ett behov för en mottagarstruktur som har förmågan att återvinna den sända signalen med så lite förvrngning som möjligt. I dagens system delas därför bredbandskanalen upp i flera icke-störande smalbandiga kanaler, som dämpar signalen jämnt. En teknisk term för denna typen av system är OFDM ("orthogonal frequency-division multiplexing"). Men med massiv MIMO leder ansamlingen av ett stort antal oberoende slumpmässiga källor ofta till en makroskopisk bild som verkar deterministisk. Detta kallas kanalhärdning. Vi undersöker huruvida denna härdande effekt förenklar den bearbetning som krävs utan att behöva dela upp den bredbandiga kanalen i flera ickeinterfererande smalbandiga kanaler, det vill säga, utan att använda OFDM. Effekten av fasbrus i bredbandiga kanaler studeras även när en viss enkel linjär behandlingstekniker används. Eftersom det finns fler antennelement på basstationen undersöks det om det är mer fördelaktigt att använda en gemensam lokal oscillator eller flera oberoende lokala oscillatorer. Massiv MIMO kräver sidoinformation om utbredningskanalen för att på lämplig sätt kombinera de mottagna signalerna vid basstationen. I massiv MIMO förvärvas denna sidoinformation med hjälp av förutbestämda signaler som kallas piloter. Fasbruset roterar dock den mottagna signalen vilket leder till en snedvridning av de mottagna piloterna. De grundläggande gränserna för kvaliteten på sidoinformationen förvärvad via piloter i Massive MIMO, när fasbrus är närvarande, undersöks också. Slutligen studeras effekten av fasbruset på massiv MIMO med OFDM. 


\section{Acknowledgments}

During the period of my doctoral studies there have been many people that played key role in my development. First and foremost, I would like to express my deepest gratitude to my main supervisor, Prof. Erik G. Larsson, for his support and supervision during all this period. His persistence to work on well-defined and important problems was pivotal to the quality and impact of my research. His meticulous review of the solutions that I was proposing was constantly transforming and improving my output and was helping me identify new directions and potential contributions.

I am also heavily indebted to Dr. Saif Khan Mohammed, now Assistant Professor in IIT Delhi, who served as co-advisor for the first years of my doctoral studies. He has been tireless in explaining various problems of my proposed solutions and in clarifying fundamental, but difficult to grasp, notions from Information Theory. He has also been very persistent to teach me how I should express my ideas in my articles clearly and effectively.

During the last years of my doctoral studies Associate Professor Dr. Emil Björnson served as my co-advisor, to whom I have to extend my genuine appreciation for his help. His feedback has been immediate, concise and accurate in every issue that was coming up. His remarkable effectiveness helped me proceed forward and acquire a broader perspective of my research area. He has also assisted me to further improve my writing style.

At the same time, I had the opportunity to work in very competitive environment with many other knowledgeable and helpful senior researchers and professors, such as Mikael Olofsson and Danyo Danev. A special thanks should be extended to Dr. Eleftherios Karipidis, now with Ericsson Research, who has helped me both with his technical knowledge and expertise but also with his personal advice. I am also grateful to Prof. Michail Matthaiou, Queen's University, Belfast, UK, for his continuing support and useful advice since my master's studies in TU Munich. Among my doctoral colleagues I would like to express my special gratitude to Dr. Hien 
Quoc Ngo, with whom I have spent many hours discussing solutions on problems and clarifying the details of his work, which helped my research efforts. Finally, many thanks to all the other colleagues that have passed from the corridor of Communication Systems and have enlightened me with their presentations and during personal discussions.

Last but not least, I would like to thank my parents and my sister as silent and indirect "co-authors" of this dissertation. Their contribution was their support and care for all my life and the opportunity they gave me to work in a peaceful and comfortable environment free of distractions and worries.

Linköping, April 2016 Antonios Pitarokoilis 


\section{Abbreviations}

$\begin{array}{ll}\text { AGC } & \text { Automatic Gain Control } \\ \text { AWGN } & \text { Additive White Gaussian Noise } \\ \text { BER } & \text { Bit Error Rate } \\ \text { BS } & \text { Base Station } \\ \text { CC } & \text { Constant Channel } \\ \text { CIR } & \text { Channel Impulse Response } \\ \text { CSI } & \text { Channel State Information } \\ \text { dB } & \text { decibel } \\ \text { DFT } & \text { Discrete Fourier Transform } \\ \text { DL } & \text { Downlink } \\ \text { DMC } & \text { Discrete Memoryless Channel } \\ \text { EVM } & \text { Error Vector Magnitude } \\ \text { FC } & \text { Fading Channel } \\ \text { FFT } & \text { Fast Fourier Transform } \\ \text { FIR } & \text { Finite Impulse Response } \\ \text { GBC } & \text { Gaussian Broadcast Channel } \\ \text { IBI } & \text { Inter-Block Interference } \\ \text { IEEE } & \text { Institute of Electrical and Electronics Engineers } \\ \text { i.i.d. } & \text { Independent and Identically Distributed } \\ \text { ISF } & \text { Impulse Sensitivity Function } \\ \text { ISI } & \text { Inter-Symbol Interference } \\ \text { LLR } & \text { Log-Likelihood Ratio } \\ \text { LMMSE } & \text { Linear Minimum Mean Square Error } \\ \text { LO } & \text { Local Oscillator } \\ \text { LoS } & \text { Line-of-Sight } \\ \text { LPF } & \text { Low-Pass Filter } \\ \text { LTI } & \text { Linear Time Invariant } \\ \text { MAP } & \text { Maximum A Posteriori } \\ \text { MIMO } & \text { Multiple-Input Multiple-Output } \\ \text { MISO } & \text { Multiple-Input Single-Output } \\ & \end{array}$


ML

MMSE

MRC

MRT

MU

MUI

NS

OFDM

OFDMA

PAM

PAPR

PDP

PLL

PN

PSK

QAM

QPSK

RF

RZF

$S$

SBS

SER

SI

SIMO

SINR

SISO

SNR

SU

TDD

TR

UL

UNF

UT

WLAN

ZF
Maximum Likelihood

Minimum Mean Square Error

Maximum Ratio Combining

Maximum Ratio Transmission

Multiuser

Multiuser Interference

Non-Synchronous

Orthogonal Frequency Division Multiplexing

Orthogonal Frequency Division Multiple Access

Pulse Amplitude Modulation

Peak-to-Average-Power-Ratio

Power Delay Profile

Phase-Lock Loop

Phase Noise

Phase Shift Keying

Quadrature Amplitude Modulation

Quadrature Phase Shift Keying

Radio Frequency

Regularized Zero-Forcing

Synchronous

Symbol-by-Symbol

Symbol Error Rate

Side Information

Single-Input Multiple-Output

Signal-to-Interference-plus-Noise Ratio

Single-Input Single-Output

Signal-to-Noise Ratio

Single-User

Time Division Duplex

Time-Reversal

Uplink

Use-And-Forget

User Terminal

Wireless Local Area Network

Zero-Forcing 


\section{Contents}

Abstract $\mathbf{v}$

Populärvetenskaplig Sammanfattning (in Swedish) vii

Acknowledgments ix

Abbreviations $\quad$ xi

I Introduction $\quad 1$

1 Motivation 3

2 Phase Noise in RF Oscillators $\quad 7$

2.1 Macroscopic Manifestation of Phase Noise . . . . . . . 7

2.2 Phase Noise Sources . . . . . . . . . . . . . . . . . 9

2.3 Circuit-theoretic Modeling of Phase Noise . . . . . . . . . . . 11

2.3.1 The Model by Leeson [25] . . . . . . . . . . . . . . . 12

2.3.2 The Model by Hajimiri and Lee [13] . . . . . . . . . . 13

2.3.3 The Model by Demir, et al. [14] . . . . . . . . . . . . 14

3 Phase Noise in Communication Systems $\quad 17$

3.1 The Complex Baseband Representation in the Presence of Phase Deviations . . . . . . . . . . . . . . 17

3.2 The AWGN Channel Impaired with Phase Noise . . . . . . . 19

3.3 The Band-Limited Phase Noise Channel . . . . . . . . . . . . 21

3.4 The Wiener Phase Noise Model in Communications . . . . . 24

4 Massive MIMO with Phase Noise Impairments 27

4.1 The MU-MIMO Uplink Channel . . . . . . . . . . . . . . 27

4.2 Massive MIMO . . . . . . . . . . . . . . . . . . . . . . . . . 29

4.3 Massive MIMO with Phase Noise . . . . . . . . . . . . . . . 31

4.3 .1 Uplink Training . . . . . . . . . . . . . . . . . . . 32

4.3.2 Data Transmission . . . . . . . . . . . . . . 32

4.4 Alternative Capacity Bound for Massive MIMO with Phase Noise . . . . . . . . . . . . . . . . . 35 
5 Contributions of the Dissertation 41

5.1 Included Papers . . . . . . . . . . . . . . . . . . . . 41

5.2 Not Included Papers . . . . . . . . . . . . . . . . . . . 45

5.3 Future Research Directions . . . . . . . . . . . . . . . . . . 45

II Included Papers $\quad 59$

A On the Optimality of Single-Carrier Transmission in Large$\begin{array}{ll}\text { Scale Antenna Systems } & 61\end{array}$

1 Introduction . . . . . . . . . . . . . . . . . . 64

2 System Model . . . . . . . . . . . . . . . . . . . . . . 65

3 Achievable Sum-Rate . . . . . . . . . . . . . . . . 66

4 Simulation Results . . . . . . . . . . . . . . . . . 71

B Uplink Performance of Time-Reversal MRC in Massive MIMO $\begin{array}{ll}\text { Systems Subject to Phase Noise } & 77\end{array}$

1 Introduction . . . . . . . . . . . . . . 80

2 System Model . . . . . . . . . . . . . . . . . . . . . . . . . 82

$2.1 \quad$ Phase Noise Model . . . . . . . . . . . . . . . . . . . . 83

2.2 Received Signal . . . . . . . . . . . . . . . . . . . 84

3 Transmission Scheme and Receive Processing . . . . . . . . . 84

3.1 Channel Estimation . . . . . . . . . . . . 85

3.2 Time-Reversal Maximum Ratio Combining (TR-MRC) 86

4 Achievable Sum-Rate . . . . . . . . . . . . . . . . . . . 87

4.1 Exact Analysis of Synchronous versus NonSynchronous Operation for a Toy Channel Model . . 94

5 Asymptotic Results . . . . . . . . . . . . . . . . . . . 95

6 Impact of Phase Noise Separately at the BS and at the User Terminals . . . . . . . . . . . . . . . . . . 99 97

6.1 Special Case 1: Phase Noise Only at the UTs, $\sigma_{\phi}^{2}=0.97$

6.2 Special Case 2: Phase Noise Only at the BS, $\left(\sigma_{\phi}^{2} \neq 0\right.$ and $\left.\sigma_{\theta}^{2}=0\right) \ldots \ldots \ldots \ldots \ldots$

$7 \quad$ Numerical Examples . . . . . . . . . . . . . . . . . . . . 99

8 Conclusions . . . . . . . . . . . . . . . . . 105

C Achievable Rates of ZF Receivers in Massive MIMO with Phase $\begin{array}{ll}\text { Noise Impairments } & 117\end{array}$

1 Introduction . . . . . . . . . . . . . . . . . . 120

2 System Model . . . . . . . . . . . . . . . . . . . . . . . . . 121

3 Transmission Scheme and ZF Receiver . . . . . . . . . . . . . 122 
3.1 LMMSE Channel Estimation . . . . . . . . . . . 123

3.2 Zero-Forcing (ZF) Equalization . . . . . . . . . . . . . 124

4 Achievable Rates . . . . . . . . . . . . . . . . . . . . 125

5 Results-Discussion . . . . . . . . . . . . . . . . . 128

D ML Detection in Phase Noise Impaired SIMO Channels with $\begin{array}{ll}\text { Uplink Training } & 135\end{array}$

1 Introduction . . . . . . . . . . . . . . . . . . . . . . . . . 138

2 System Model . . . . . . . . . . . . . . . . . . . . . . . . 141

2.1 Constant Channel (CC) . . . . . . . . . . . . . . 142

2.2 Fading Channel (FC) . . . . . . . . . . . . . . . 143

3 Optimal Detectors . . . . . . . . . . . . . . . . 144

3.1 Implementation of (15) and (25) . . . . . . . . . 148

3.2 The ML Detector for von Mises Phase Noise Increments148

4 High SNR Analysis . . . . . . . . . . . . . . . . . . 150

4.1 High SNR Analysis for the Synchronous Operation . 150

4.2 High SNR Analysis for the CC-NS Case . . . . . . . . 152

4.3 High SNR Analysis for the FC-NS Case . . . . . . . 154

5 Numerical Examples . . . . . . . . . . . . . . . . . . 156

5.1 Extension to Longer Data Intervals . . . . . . . . . . 161

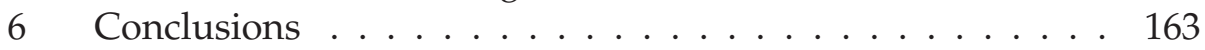

7 Two-Slot Proofs . . . . . . . . . . . . . . . . . . . 165

7.1 Proof of Proposition 1 for the CC-NS Case . . . . . 165

7.2 Proof of Proposition 1 for the FC-NS Case . . . . . . 167

7.3 Proof of Proposition 2 for the CC-S Case . . . . . . . . 169

7.4 Proof of Proposition 2 for the FC-S Case . . . . . . . . 169

7.5 Proof of Proposition 3 . . . . . . . . . . . . . . . . 169

8 T-slot Detectors . . . . . . . . . . . . . . . . . . . 170

8.1 Decision Feedback Detector: CC-NS . . . . . . . . . 170

8.2 "Better-than-optimal" Detector: CC-S . . . . . . . . . 172

8.3 Decision Feedback Detector: FC-NS . . . . . . . . 173

8.4 "Better-than-optimal" Detector: FC-S . . . . . . . . . 175

8.5 Numerical Examples . . . . . . . . . . . . . . 176

E Performance of the Massive MIMO Uplink with OFDM and $\begin{array}{ll}\text { Phase Noise } & 185\end{array}$

1 Introduction . . . . . . . . . . . . . . . . . . . . . 188

2 System Model . . . . . . . . . . . . . . . . . . . . . . . . . . . 189

3 Achievable Rates . . . . . . . . . . . . . . . . . . . . . . . 191

4 Numerical Examples . . . . . . . . . . . . . . . . . . . . 194

5 Conclusion . . . . . . . . . . . . . . . . . 196 



\section{Part I}

\section{Introduction}





\section{Chapter 1}

\section{Motivation}

Arguably, the world has experienced a rapid growth of data traffic over wireless networks in the last decades. It is reported that the global mobile data traffic has increased by more than 400 million times over the past 15 years, from less than $10 \mathrm{~GB}$ per month in 2000 to $3.7 \mathrm{~EB}^{1}$ per month at the end of 2015 [1]. This trend is not expected to cease any time soon. In fact it is predicted that it will accelerate in the years to come, as the traffic is expected to reach $30 \mathrm{~EB}$ per month by 2020 [1]. This demand is expected to originate not only from wireless data exchange by tablets and smartphones but also from the proliferation of new types of communication, such as machineto-machine communication [2]. In short, any device that could collect and disseminate information via the wireless medium and could benefit from this data exchange, is expected to be equipped with wireless communication capabilities.

The straightforward way to satisfy the increase in traffic demand is to increase the frequency spectrum for the communication. However, there are various reasons that make this approach not attractive. First, the spectrum is a constrained natural resource. Within a fixed spectrum portion multiple communication services, military and civilian, must be accommodated. Already, most part of the available spectrum is allocated to various services and operators, so if we are to allocate more spectrum to mobile communications, spectrum portions from other services must be made available. This might be possible, but only to a very restricted extent, which is definitely not enough to fulfill the demand for the future mobile data traffic.

\footnotetext{
${ }^{1} 1 \mathrm{~EB}$ (ExaByte) is 1 billion $\left(10^{9}\right) \mathrm{GB}$ (GigaByte).
} 
Also, certain frequency spectrum bands are not appropriate for wireless networks, due to unfavorable propagation conditions or high atmospheric attenuation. Finally, spectrum is a very expensive resource for mobile operators. It is clear that a more aggressive and spectrally efficient approach must be applied for the evolution of wireless networks. A more aggressive spectrum reuse can increase data rates within certain geographical areas of interest. This can be partly implemented with the deployment of small cells (including WiFi access points). However, small cells are less effective when dealing with high-mobility users and providing wide-area coverage.

The deployment of multiple antennas at the transmitter and receiver (multiple-input multiple-output (MIMO)) was shown to provide significant gains in the amount information that can be communicated within a fixed frequency band. The gains scale linearly with the minimum of the transmit and receive antennas in the presence of rich scattering environment and when the channel is known at the receiver [3,4]. Simultaneously with the emergence of point-to-point MIMO, the concept of multi-user MIMO (MU-MIMO) was investigated, where a base station (BS) equipped with a handful of antennas communicates with a few non-cooperative users over the same time and frequency resource [5]. Early work on the topic includes [6-8]. Point-to-point MIMO provide the promised gains in rich scattering scenarios, but require large antenna separation in the presence of a strong Line-of-Sight (LoS) component to achieve the same performance. In contrast, MU-MIMO are more robust in LoS conditions. However, the adoption of MU-MIMO techniques in modern wireless communication standards has not been proportional to the available research literature due to the fact that the optimal transmission strategies are complex and require accurate channel state information (CSI) at the BS.

Massive MIMO [9], also known as Large Scale Antenna Systems, Very Large MIMO, Full-Dimension MIMO, proposes a new approach towards the practical implementation of ideas from MU-MIMO, where a BS with an unprecedentedly large number of BS antenna elements, $M$, serves simultaneously a few tens of single-antenna, non-cooperative users, $K$. When $M \gg K$ linear, low-complexity processing techniques are close to optimal, while at the same time, channel state acquisition can be made available at the BS via uplink training. This way the resources spent for channel training are proportional to the number of users and, thus, the design is scalable with $M$. Massive MIMO is shown to achieve substantial gains in spectral and radiated energy efficiency of future wireless networks [10,11]. 
Massive MIMO is not just an increase of the number of antenna elements at the BS and a corresponding adoption of linear processing strategies. This newly proposed physical layer can also have further implications on the design of wireless networks. Currently, most wireless standards use multicarrier techniques to transmit data over wireless channels. Even though multicarrier transmission is very likely to be part of next generation wireless networks, it would be useful to investigate whether with Massive MIMO there exist regimes or deployment scenarios where low complexity single-carrier transmission can provide comparable or even superior performance than currently used techniques. In particular, with single-carrier transmission low peak-to-average-ratio (PAPR) waveforms can be designed. With such waveforms, low quality, inexpensive, power efficient, non-linear power amplifiers can be used at each BS antenna element, which is important in Massive MIMO. In current systems expensive, power inefficient, highly linear amplifiers with a large power back-off are required to transmit signals with high PAPR. Multi-carrier signals are known to have a large PAPR. With single-carrier transmission, all the available bandwidth is allocated to a user, which simplifies the user scheduling. Further, the transmission when $M \gg K$ will be robust against intersymbol interference. Finally, single-carrier transmission is less sensitive to impairments, such as carrier phase instabilities, in comparison to multi-carrier techniques, such as orthogonal frequency division multiplexing (OFDM).

The deployment of economically viable Massive MIMO relies on the use of multiple inexpensive components that are likely to introduce distortions to the information signal. Hence, affordable Massive MIMO must be robust against hardware impairments that arise due to imperfections in the transmitter and the receiver circuits. Therefore, the study of the effect of hardware impairments, which is frequently treated as secondary in the study of conventional systems, is very important in Massive MIMO. One of the most important hardware impairments is phase noise, which is introduced in communication systems due to imperfections in the circuits of the local oscillators (LOs) at the transmitter and receiver chains. Imperfect LOs introduce random and time-varying rotations at the information signals. These rotations hinder the receiver to make the correct decisions about the transmitted signal based on the available noisy observations. Phase noise affects not only the data detection, but also the channel estimation. As Massive MIMO relies on the coherent combining of the received signals with estimated channel impulse responses in order to provide the promised gains, phase noise destroys the coherency between the channel estimates and the 
effective channels at the time of decoding.

In this dissertation we investigate the performance of Massive MIMO with wideband transmission -single carrier and multi-carrier- and in the presence of phase noise impairments. Capacity lower bounds are derived for various scenarios of practical interest and scaling laws with respect to radiated energy efficiency and other performance measures of interest are stated. The effect of phase noise on detection with training based CSI is also studied rigorously by deriving the maximum likelihood (ML) detector. The introduction of the dissertation is structured as follows. In Chapter 2 the fundamental phenomena that give rise to phase noise in radio frequency oscillators are reviewed and the most important models are stated. In Chapter 3 the way that phase noise enters in communication systems is analyzed. In Chapter 4 the concept of Massive MIMO is briefly introduced and the basic steps for the analysis of phase noise impaired Massive MIMO systems are explained. In Chapter 5 the basic contributions of the dissertation are described and the included papers are listed. 


\section{Chapter 2}

\section{Phase Noise in RF Oscillators}

In this chapter the issue of phase noise in radio frequency (RF) LOs is discussed. The purpose is to relate the models used in this dissertation and in communications literature, in general, to well-known, established models that correspond to the physical reality of contemporary LOs. The discussion will be concentrated on free-running LOs, which is the main assumption on the oscillator operation throughout the dissertation. Initially, the macroscopic effects of phase noise on periodic waveforms are described. The main noise sources in electronic circuits that eventually perturb the waveform at the output of an LO are briefly reviewed. Finally, three main models that have been widely used to explain the behavior of phase noise are described.

\subsection{Macroscopic Manifestation of Phase Noise}

The LOs are electronic circuits that are designed to produce an oscillating waveform with a specified angular frequency, $\omega_{c}$. They are essential in wireless communications, since they are used to modulate the baseband signal to passband at the transmitter and to demodulate the received passband signal to baseband at the receiver. Ideally, the output voltage, $V_{\text {out }}(t)$, of a noiseless LO is a sinusoidal waveform that is perfectly stable in amplitude, frequency and phase, given by

$$
V_{\text {out }}(t)=A \cos \left(\omega_{c} t+\phi\right) \text {. }
$$


However, the electronic components (active and passive) that are used to manufacture the LO circuits are impaired by noise. Consequently, the output of a real LO has amplitude and phase that are time-varying and the output voltage is given by

$$
V_{\text {out }}(t)=(1+a(t)) \cos \left(\omega_{c}(t+\alpha(t))\right),
$$

where $a(t)$ is the amplitude perturbation and $\alpha(t)$ is the timing noise. Due to the amplitude limiting mechanism that is present in practical LOs, e.g., as an automatic gain control (AGC) component [12], the amplitude perturbation, $a(t)$, gradually decays. Hence, the amplitude can be assumed to be constant. On the other hand, it has been shown that the noise $\alpha(t)$ persists and gives rise to a sequence of non-vanishing, random and varying timing offsets $[13,14]$.

In the time domain, phase noise becomes apparent by observing the zerocrossings of the oscillating waveform. The time interval between two consecutive zero-crossings from positive to negative values in this oscillating waveform, which is usually the voltage or the current at the output of the $\mathrm{LO}$, should be exactly equal to the period of the LO waveform. However, in the presence of phase noise, these zero-crossings are slightly shifted, as shown in Fig. 2.1a. In the frequency domain, the power spectral density of the LO output is a Dirac impulse located at $\omega_{c}$. In the presence of phase noise, however, the power spectral density of the LO output, assuming that it is wide-sense stationary, widens around the oscillation angular frequency, $\omega_{c}$ (Fig. 2.1b).

The effect of phase noise in digital modulation is a random phase rotation of the constellation points. In Fig. 2.2 the received symbols from a 16-QAM constellation [15] are plotted in the presence of phase noise when there is no additive thermal noise. The phase deviations in this figure are assumed to be zero-mean Gaussian ${ }^{1}$ random variables with variance $10^{-2} \mathrm{rad}^{2} /$ sample. It can be seen that the received symbols can be substantially rotated by phase noise. Consequently, the probability that a received signal is detected in error is high even when the desired signal power is significantly larger than the thermal noise power $[16,17]$. The degradation due to phase noise is more detrimental for dense constellations, that convey information not only on the amplitude but also on the phase of the transmitted symbol.

Phase noise can introduce distortion not only to the transmitted/received signal but also to signals in adjacent frequency bands. At the receiver

\footnotetext{
${ }^{1}$ The justification of this choice is deferred to Section 2.3.3 and Section 3.4.
} 


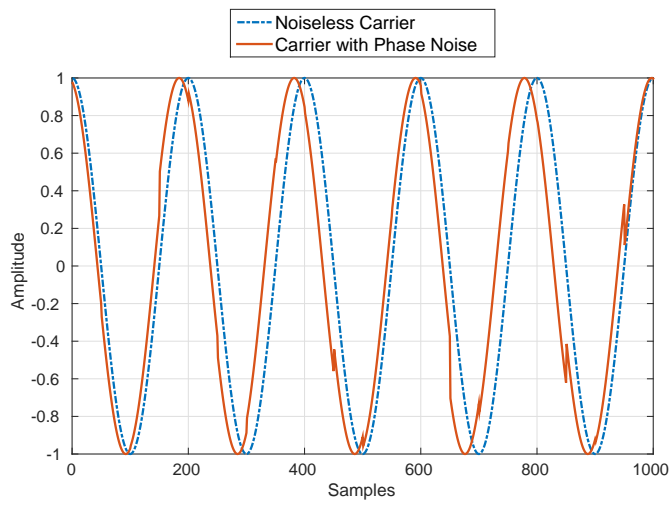

(a) Phase noise in the time domain. The zerocrossings are shifted randomly from the nominal position in the presence of phase noise.

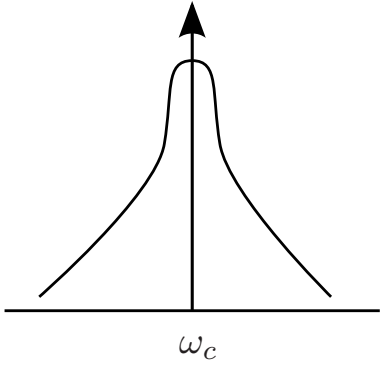

(b) Phase noise in the frequency domain. The ideal frequency spectrum widens due to phase noise.

Figure 2.1: Time-domain and frequency-domain manifestation of the output voltage from an $\mathrm{LO}$ with phase noise.

side, consider the demodulation of a weak passband signal with center frequency $\omega_{1}$, in the presence of a strong interferer in an adjacent frequency band centered at $\omega_{2}$, as shown in Fig. 2.3a. In the case of a noisy LO, significant interference can be introduced to the desired signal at $\omega_{1}$, from the strong interferer at $\omega_{2}$, shown as the shaded spectrum in Fig. 2.3b. This phenomenon is called reciprocal mixing [18]. At the transmitter side, a strong signal with a noisy $\mathrm{LO}$ at $\omega_{2}$, can introduce interference leakage to a weak neighboring signal at $\omega_{1}$ (Fig. 2.4). Since the frequency bands around $\omega_{1}$ and $\omega_{2}$ are adjacent, the difference between the two carrier frequencies can be of the order of a few $\mathrm{kHz}$. This imposes strict requirements so that the power spectral density of the noisy LOs should decay very sharply [18].

\subsection{Phase Noise Sources}

Noise is unavoidable in electronic circuits. Various random microscopic events that occur within a circuit and its components give rise to fluctuations and disturbances of the current and the voltage at the output of the circuit. The random motion of electrons within a resistor result in a voltage, 


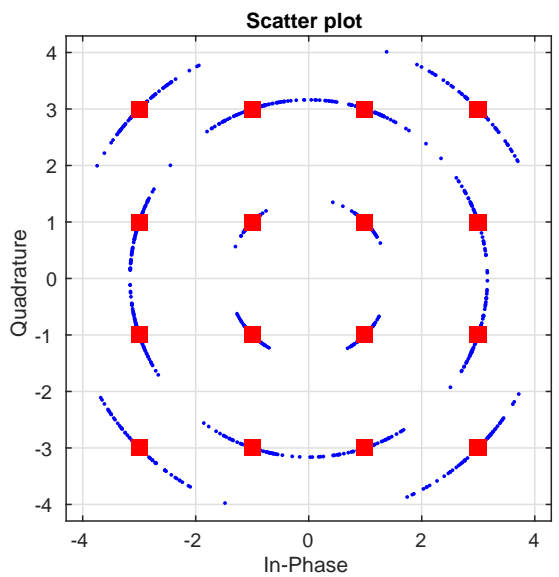

Figure 2.2: 16-QAM constellation rotation in the presence of phase noise and the absence of additive thermal noise.

$e_{n}(t)$, along the resistor that has a non-zero mean-square value even in the absence of current flow through the resistor [19]. A resistor of $R \mathrm{Ohm}$ has a mean square noise density of

$$
\overline{e_{n}^{2}}=4 k T R \Delta f,
$$

over a bandwidth of $\Delta f \mathrm{~Hz}$, where $k \approx 1.38 \times 10^{-23}$ Joule/Kelvin is the Boltzmann constant and $T$ is the temperature measured in Kelvin. This type of noise is referred to as thermal noise due to its dependence on the absolute temperature, $T$. The mean square noise density is independent of the frequency, $f$, for a very wide spectrum of frequencies, which implies that thermal noise is white for most of the frequencies of interest. Thermal noise is also known as Johnson-Nyquist noise, due to the scientists that first measured and explained the phenomenon [20,21].

Shot noise appears in devices such as diodes or bipolar transistors in the presence of a constant average current flow of $I_{\mathrm{DC}}$ Ampère. These devices have a potential barrier that the charge carriers have to cross [19]. Due to the discrete type of the microscopic electron motions and the randomness in the time instants when these motions occur, fluctuations around $I_{\mathrm{DC}}$ appear. A shot noise source has a mean square current noise at a bandwidth of $\Delta f \mathrm{~Hz}$ given by

$$
\overline{i_{n}^{2}}=2 q I_{\mathrm{DC}} \Delta f,
$$




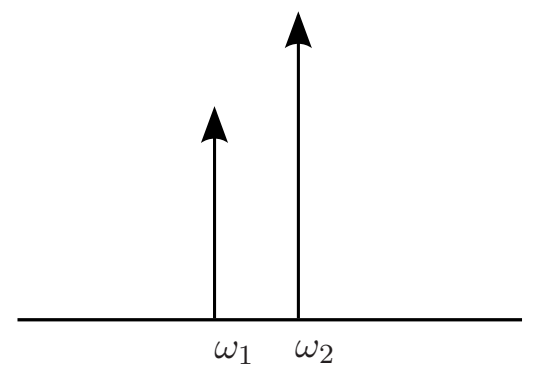

(a) Weak desired signal at $\omega_{1}$, in the presence of strong signal in an adjacent frequency band centered at $\omega_{2}$.

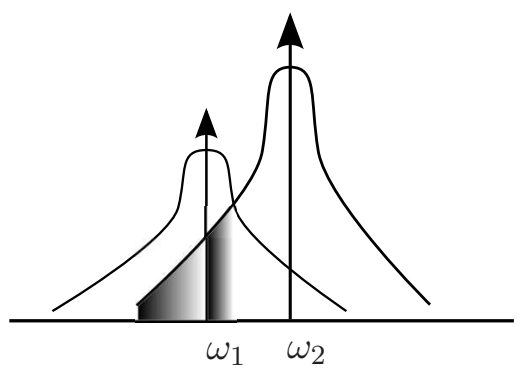

(b) Spectral widening due to noisy $\mathrm{LO}$ at the receiver. Strong interference from the adjacent signal at $\omega_{2}$, leaks into the bandwidth of the desired signal at $\omega_{1}$.

Figure 2.3: Reciprocal mixing due to noisy LO at the receiver [18].

where $q \approx 1.6 \times 10^{-19}$ Coulomb is the electron charge. The mean square current noise $\overline{i_{n}^{2}}$ is independent of the frequency, $f$, which implies that shot noise is also white. The description and explanation of shot noise is attributed to W. Schottky [22].

Another type of noise that is present in physical systems is flicker noise. It has been observed in the fluctuations of various physical phenomena, spanning from biology, to electronics and astrophysics. This type of noise is colored and has power spectral density that scales inverse-proportionally with the frequency. For this reason, flicker noise is also called $1 / f$-noise $[23,24]$. The physical mechanism that generates flicker noise is still debatable in the physics community and its description involves empirical parameters [19].

\subsection{Circuit-theoretic Modeling of Phase Noise}

In this section we briefly review three of the most important models that have been used to explain the behavior of the output of noisy LOs. The models are presented in the chronological order that they appeared and the main contribution on the understanding and modeling of phase noise is concisely explained. 


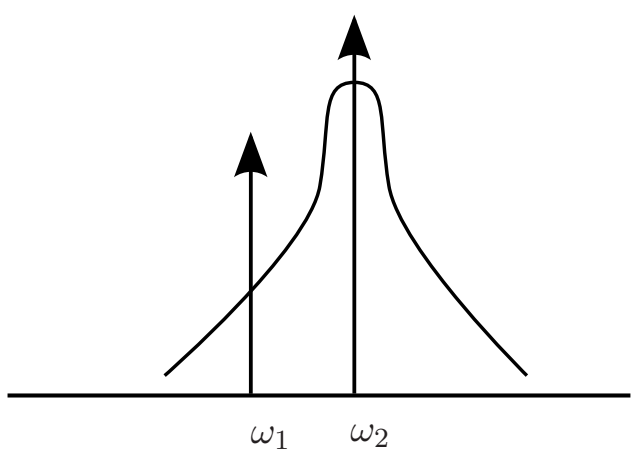

Figure 2.4: Interference leakage at the transmitter due to strong signal with high phase noise spectrum at $\omega_{2}$ in the bandwidth of the desired signal at $\omega_{1}$.

\subsubsection{The Model by Leeson [25]}

The most common measure of the output of a noisy LO, usually a voltage or a current, is the single-sided power spectral density defined by [13]

$$
\mathcal{L}(\Delta \omega) \triangleq 10 \log _{10}\left[\frac{\mathcal{P}\left(\omega_{c}+\Delta \omega, 1 \mathrm{~Hz}\right)}{\mathcal{P}_{c}}\right],
$$

where $\mathcal{P}\left(\omega_{c}+\Delta \omega, 1 \mathrm{~Hz}\right)$ is the single-sided power measured at frequency band of width $1 \mathrm{~Hz}$ located at an offset of $\Delta \omega$ from the carrier, $\omega_{c}$, and $\mathcal{P}_{c}$ is the power of the carrier. The unit of $\mathcal{L}(\Delta \omega)$ is decibels below carrier per Hertz, $(\mathrm{dBc} / \mathrm{Hz})^{2}$ [26]. The single-sided power spectral density at the output of a noisy LO as a function of the offset, $\Delta \omega$, from the nominal oscillation frequency, $\omega_{c}$, has, in general, a shape similar to the one shown in Fig. 2.5. Three different regions are apparent, namely, i) the small offset region, where $\mathcal{L}(\Delta \omega)$ drops as $1 / f^{3}$, ii) the medium offset region, where $\mathcal{L}(\Delta \omega)$ drops as $1 / f^{2}$ and iii) the large offset region, where $\mathcal{L}(\Delta \omega)$ reaches a constant level. Leeson in 1966 [25] attempted to explain this spectrum using linear time-invariant system theory. The derived spectrum is given by

$$
\mathcal{L}(\Delta \omega)=10 \log _{10}\left[\frac{2 F k T}{P_{\text {sig }}}\left\{1+\left(\frac{\omega_{c}}{2 Q \Delta \omega}\right)^{2}\right\}\left(1+\frac{\Delta \omega_{1 / f^{3}}}{|\Delta \omega|}\right)\right],
$$

\footnotetext{
${ }^{2}$ The careful reader will observe that the "per Hertz" should apply to the quantities inside the logarithm. However, in practice the unit is used as here, with the implicit understanding that $\mathrm{Hz}$ should refer to the argument of the logarithm rather than the logarithm itself.
} 


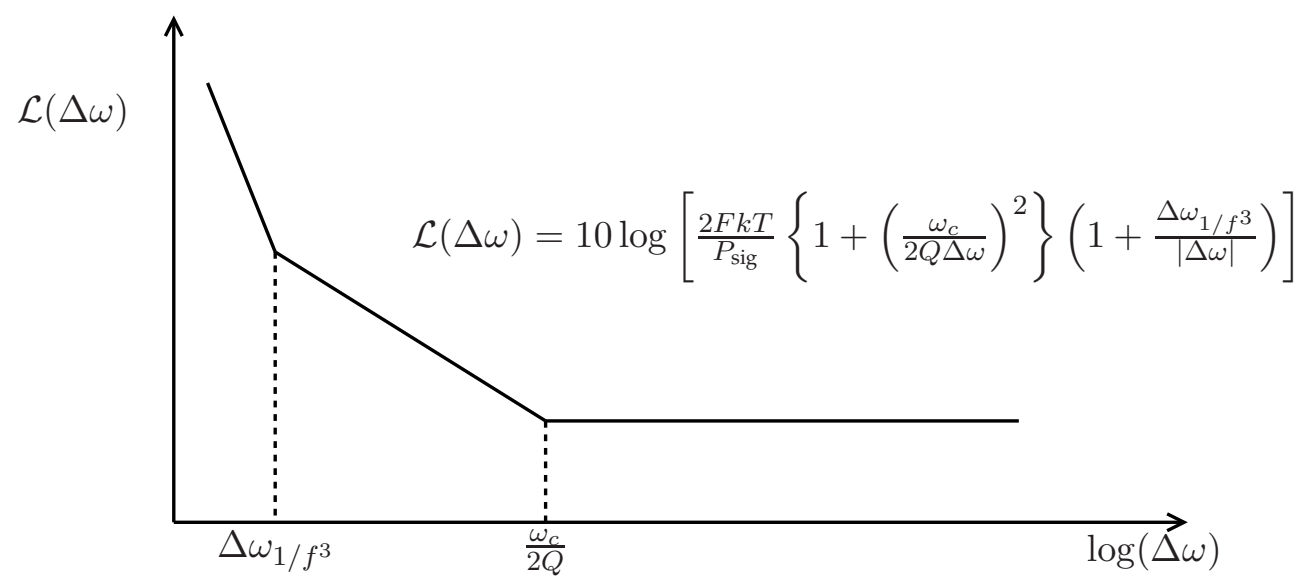

Figure 2.5: Single-sided power spectral density of a noisy LO output as predicted by Leeson [25].

where $\Delta \omega$ is the frequency offset from the carrier, $k$ is the Boltzmann constant, $T$ is the temperature in Kelvin, $P_{\text {sig }}$ is the signal power, $Q$ is the resonator $^{3}, F$ is the device excess noise number [13] and $\Delta \omega_{1 / f^{3}}$ is the corner frequency offset between the $1 / f^{3}$ region and the $1 / f^{2}$ region. Apart from matching the predicted spectrum at the output of a noisy LO, Leeson's model also reveals some useful rules of thumb. For instance, an LO with high $Q$, i.e., good oscillator, will have a better phase noise performance. Leeson's model, despite its heuristic nature, has been a major reference for many years.

\subsubsection{The Model by Hajimiri and Lee [13]}

Leeson's model has certain drawbacks. For instance, $F$ is an empirical parameter that cannot be predicted before the design of the LO. Further, the value of $\Delta \omega_{1 / f^{3}}$ in (2.6) must also be determined empirically in practice. The reason for these deviations from reality is the fact that LOs are neither linear nor time-invariant systems. Hence, the Leeson approach, which relied on these assumptions, did not properly reflect the physical reality.

Hajimiri and Lee $[13,26]$ showed that LOs are time-varying systems and dropped the time-invariance assumption. They represented the noise

\footnotetext{
${ }^{3}$ This is a parameter that shows the quality of the oscillator and is defined as the ratio of the energy stored in the oscillator's reactive elements over the energy dissipated [19].
} 
sources in a general LO as current impulses and they argued that the resulting perturbations on the LO output can be decomposed to pure amplitude and pure phase perturbations. While the amplitude perturbations go to zero due to the amplitude limiting mechanisms in the circuit, the phase perturbations do not. Further, they argued that the impulse response of the varying phase, $\phi(t)$, at the LO output to a current noise impulse (currentto-phase transfer function) is still linear but time-varying. They introduced special functions that they called impulse sensitivity functions (ISFs), which contain information on the sensitivity of the output $\mathrm{LO}$ waveform to circuit noise impulses. In general, ISFs depend on the oscillating waveform and are usually determined numerically. The authors used the theory of linear time-varying systems to derive the spectrum at the output of a noisy LO. The derived model gives a more accurate and less heuristic explanation of the power spectral density of a noisy LO output. Therefore, it helps designers identify the dominant sources of phase noise and take them into consideration in their design.

\subsubsection{The Model by Demir, et al. [14]}

The models in Sections 2.3.1 and 2.3.2 explain the phenomenon of phase noise in terms of the output power spectral density rather than the timedomain statistical characterization of the phase noise process. Such a description is more useful to the designers of LOs and less to communications engineers. An asymptotic statistical characterization of the phase noise process in the time domain is given by Demir, et al. [14]. They revisited the conjecture of Hajimiri and Lee that the perturbations can be decomposed to pure amplitude and pure phase perturbations and studied LOs as dynamical systems, i.e., systems that can be described by a differential equation in the presence of some perturbation

$$
\frac{\mathrm{d}}{\mathrm{d} t} x(t)=f(x(t))+B(x(t)) b(t),
$$

where $x(t)$ is the state of the system-typically a vector of voltages and/or currents along capacitors and inductors of the LO-at time $t, f(\cdot)$ is somenot necessarily linear-function and $B(x(t)) b(t)$ is a small state-dependent perturbation. They assumed that there is a stable non-trivial periodic solution, $x_{s}(t)$, ("orbit" or "limit cycle" [27]) to the unperturbed system, i.e., (2.7) without the term $B(x(t)) b(t)$, and they showed that with a small perturbation the orbit changes to $x_{s}(t+\alpha(t))+y(t)$, where $y(t)$ is an amplitude perturbation that eventually disappears, whereas the time shift, $\alpha(t)$, 
persists. Hence, they showed that the behavior of $\alpha(t)$ demonstrates the accumulative nature of phase noise. They also proceeded to show that, asymptotically in $t$ and when the circuit noise sources are white (thermal and shot noise), the characteristic function of $\alpha(t)$ is that of a Gaussian random variable and that the autocorrelation function of the time shift $\alpha(t)$ is given by

$$
\mathbb{E}[\alpha(t) \alpha(t+\tau)]=m^{2}+c \min (t, t+\tau),
$$

where $m$ and $c$ are constants. The asymptotic statistical characterization of $\alpha(t)$ in (2.8) implies that the increments $\alpha(t)-\alpha(t+\tau)$ are Gaussian and have variance that is proportional to $|\tau|[28]$. These properties correspond to a continuous-time Brownian motion or Wiener process [29, Section 37] and justify a model that is widely used in communications, the Wiener phase noise model. The fact that phase noise can be described in the time domain with a very compact characterization that depends on a single scalar, $c$, makes the Wiener model very attractive for study of phase noise in the fields of Information Theory and Detection \& Estimation Theory. A more detailed analysis of this model in a communications setup is deferred to Section 3.4. Demir extended the analysis to include white and colored noise sources (i.e., flicker noise), however, the expressions attained are much more complicated and involve the integration over the spectra of the colored noise sources [30, Lemma 7.1]. 


\section{Chapter 3}

\section{Phase Noise in Communication Systems}

In the following the impairment of phase noise is introduced in suitable communication system models. The discussion starts with the complex baseband representation of a real passband signal as it passes through a communication system with phase deviations at the transmitter and the receiver. Subsequently, the single-input single-output (SISO) phase noise impaired additive white Gaussian noise (AWGN) channel is discussed and based on the continuous-time system model, a discrete-time approximation is derived. The band-limited SISO channel with phase noise impairments at the transmitter and the receiver is also introduced and discrete-time approximations are derived. The chapter concludes with the description of the Wiener phase noise model, which has been motivated by Section 2.3.3, and its connection to the communication-theoretic system models is described.

\subsection{The Complex Baseband Representation in the Presence of Phase Deviations}

Let two sequences $a(t)$ and $b(t)$ of pulse amplitude modulation (PAM) symbols to be transmitted over a channel located at a frequency, $f_{c}$. The channel is assumed to be noiseless and does not introduce any other distortion to 


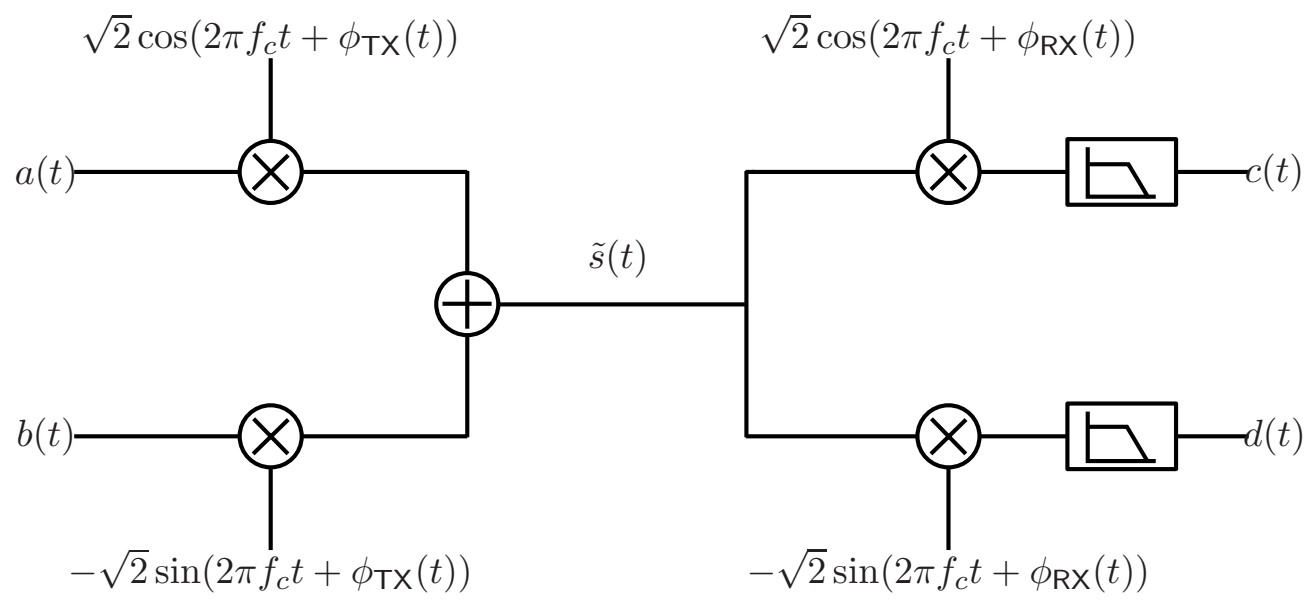

Figure 3.1: Passband representation of a system with phase deviations at the transmitter and the receiver.

the transmitted signal. However, we assume that phase noise distortions, $\phi_{\mathrm{TX}}(t)$ and $\phi_{\mathrm{RX}}(t)$, are introduced due to noisy LOs at the transmitter and the receiver, respectively. The real passband signal after the modulation is given by

$$
\begin{aligned}
\tilde{s}(t) & =a(t) \sqrt{2} \cos \left(2 \pi f_{c} t+\phi_{\mathrm{TX}}(t)\right)-b(t) \sqrt{2} \sin \left(2 \pi f_{c} t+\phi_{\mathrm{TX}}(t)\right) \\
& =\Re\left\{(a(t)+j b(t)) e^{j \phi_{\mathrm{TX}}(t)} \sqrt{2} e^{j 2 \pi f_{c} t}\right\} .
\end{aligned}
$$

Hence, the complex baseband equivalent representation of the transmitted signal in the presence of phase deviations at the transmitter is given by $s_{b}(t)=(a(t)+j b(t)) e^{j \phi_{\mathrm{TX}}(t)}$.

At the receiver, the in-phase component, $c(t)$, is given by

$$
\begin{aligned}
c(t) & =\operatorname{LPF}\left\{\tilde{s}(t) \sqrt{2} \cos \left(2 \pi f_{c} t+\phi_{\mathrm{RX}}(t)\right)\right\} \\
& =a(t) \cos \left(\phi_{\mathrm{TX}}(t)-\phi_{\mathrm{RX}}(t)\right)-b(t) \sin \left(\phi_{\mathrm{TX}}(t)-\phi_{\mathrm{RX}}(t)\right),
\end{aligned}
$$

where $\operatorname{LPF}\{\cdot\}$ is the operation of a low-pass filter, which filters out the frequency component at $2 f_{c}$. Similarly, the quadrature component is given by

$$
\begin{aligned}
d(t) & =\operatorname{LPF}\left\{\tilde{s}(t) \sqrt{2} \sin \left(2 \pi f_{c} t+\phi_{\mathrm{RX}}(t)\right)\right\} \\
& =a(t) \sin \left(\phi_{\mathrm{TX}}(t)-\phi_{\mathrm{RX}}(t)\right)+b(t) \cos \left(\phi_{\mathrm{TX}}(t)-\phi_{\mathrm{RX}}(t)\right) .
\end{aligned}
$$


The received complex baseband signal is

$$
y(t)=c(t)+j d(t)=(a(t)+j b(t)) e^{j \phi_{\mathrm{TX}}(t)} e^{-j \phi_{\mathrm{RX}}(t)} .
$$

In the case of narrowband SISO systems, the baseband equivalent representation in (3.4) reduces to $y(t)=(a(t)+j b(t)) e^{j \theta(t)}$, where only the difference $\theta(t)=\phi_{\mathrm{TX}}(t)-\phi_{\mathrm{RX}}(t)$ is important. However, in MU-MIMO systems the distinction between the transmit and receive phase deviations is essential. Consider for example a system with two non-cooperative single-antenna transmitters and a single-antenna receiver. The two effective channels from the transmitters to the receiver will be both affected by the same receive phase noise process but from independent transmit phase noise processes. This must be reflected by the selected channel representation.

\subsection{The AWGN Channel Impaired with Phase Noise}

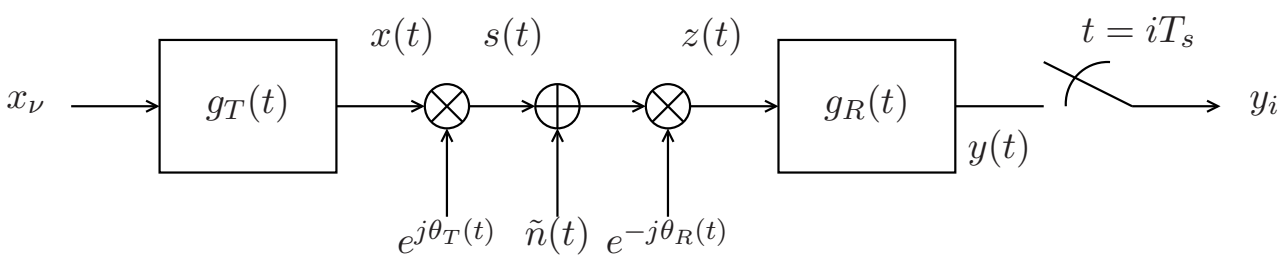

Figure 3.2: The continuous-time additive white Gaussian (AWGN) channel with phase noise.

Based on the complex baseband equivalent representation of phase noise impaired SISO systems from Section 3.1, the phase noise impaired AWGN channel is studied in continuous time and a discrete time approximation is derived. Let the waveform to be transmitted, $x(t)$, be

$$
x(t)=\sum_{\nu=1}^{N} x_{\nu} g_{T}\left(t-\nu T_{s}\right),
$$

where $T_{s}$ is the symbol interval, $\left\{x_{1}, \ldots, x_{N}\right\}$ is the sequence of transmitted symbols selected from a fixed constellation [15] and $g_{T}(t)$ is the impulse response of the transmit pulse shaping filter. The actually transmitted waveform is disturbed by the transmit phase noise process, $\theta_{T}(t)$ :

$$
s(t)=e^{j \theta_{T}(t)} x(t)=e^{j \theta_{T}(t)} \sum_{\nu=1}^{N} x_{\nu} g_{T}\left(t-\nu T_{s}\right) .
$$


The channel introduces to the transmitted signal, $s(t)$, additive noise $\tilde{n}(t)$, which is a white Gaussian random process. At the receiver the signal after the downconversion by the $\mathrm{LO}$ at the receiver, $z(t)$, is given by

$$
z(t)=\sum_{\nu=1}^{N} x_{\nu} e^{-j\left(\theta_{R}(t)-\theta_{T}(t)\right)} g_{T}\left(t-\nu T_{s}\right)+n(t) .
$$

Due to the circular symmetry of the $\tilde{n}(t)$ noise process, $n(t)=e^{-j \theta_{R}(t)} \tilde{n}(t)$ and $\tilde{n}(t)$ have the same second order statistical characterization. Further, it is clear that $\theta_{T}(t)$ and $\theta_{R}(t)$ are observed only through their difference. Hence, in what follows we note $\theta(t) \triangleq \theta_{T}(t)-\theta_{R}(t)$. At the receiver, the signal is filtered by a linear time-invariant (LTI) filter with impulse response, $g_{R}(t)$, which is matched to $g_{T}(t)$. The output of the filter $g_{R}(t)$ is given by

$$
\begin{aligned}
y(t)=\int_{-\infty}^{+\infty} g_{R}(\tau) z(t-\tau) \mathrm{d} \tau & =\sum_{\nu=1}^{N} x_{\nu} \int_{-\infty}^{+\infty} e^{j \theta(t-\tau)} g_{T}^{*}(-\tau) g_{T}\left(t-\tau-\nu T_{s}\right) \mathrm{d} \tau \\
& +\int_{-\infty}^{+\infty} g_{R}(\tau) n(t-\tau) \mathrm{d} \tau
\end{aligned}
$$

The mathematical manipulation of the derived continuous-time model appears to be formidable. In addition, the progress of digital electronics motivates the discretization of the continuous-time model in (3.8). For this purpose, the received signal, $y(t)$, is sampled at regular intervals equal to the symbol interval, $T_{s}$. The sample $y_{i}$ at time $t=i T_{s}$ is given by

$$
\begin{aligned}
y_{i} & =y\left(t=i T_{s}\right) \\
& =\sum_{\nu=1}^{N} x_{\nu} \int_{-\infty}^{+\infty} e^{j \theta\left(i T_{s}-\tau\right)} g_{T}^{*}(-\tau) g_{T}\left(i T_{s}-\tau-\nu T_{s}\right) \mathrm{d} \tau+n_{i}
\end{aligned}
$$

A common assumption is that phase noise is constant within a symbol interval, but it does change between two consecutive symbol intervals. Under this assumption and if the cascade of the transmit and receive filters $g_{T}(t)$ and $g_{R}(t)$ satisfies the Nyquist criterion for intersymbol interference [15] the following simple symbol-sampled phase noise channel model follows immediately

$$
y_{i}=x_{i} e^{j \theta_{i}}+n_{i},
$$

where the noise samples $n_{i}$ are jointly Gaussian and uncorrelated, hence statistically independent [31, Section 3.3.3]. Under these conditions, the sequence $y_{1}, \ldots, y_{N}$ is sufficient statistics for the detection of $x_{1}, \ldots, x_{N}$. The 
model (3.10) has been extensively studied under various assumptions on the statistics of the phase sequence, $\left\{\theta_{i}\right\}$. The arguments used above for the derivation of the discrete-time symbol-sampled phase noise model are in general well-known and can be found in literature either implicitly [32] or more explicitly $[33,34]$.

\subsection{The Band-Limited Phase Noise Channel}

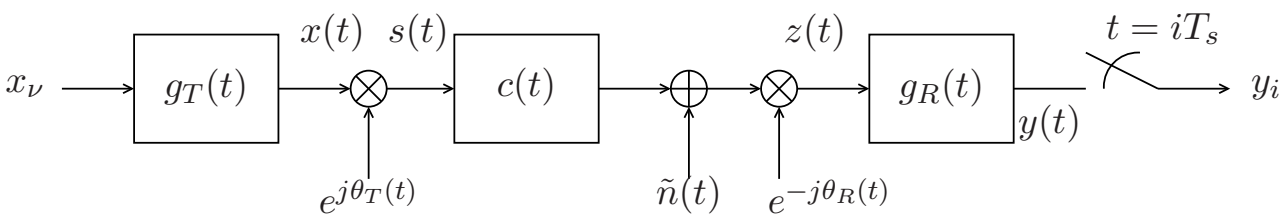

Figure 3.3: The continuous-time band-limited channel with phase noise.

Here we extend the discussion to the case of a band-limited channel with complex baseband equivalent impulse response $c(t)$, when phase noise is introduced at the transmitter and the receiver, as shown in Fig. 3.3. We assume that the signal to be transmitted is given by

$$
x(t)=\sum_{\nu=1}^{N} x_{\nu} g_{T}\left(t-\nu T_{s}\right) .
$$

The transmitter signal, which is distorted by transmit phase noise, is given by

$$
s(t)=e^{j \theta_{T}(t)} x(t)=e^{j \theta_{T}(t)} \sum_{\nu=1}^{N} x_{\nu} g_{T}\left(t-\nu T_{s}\right) .
$$

The received signal after the filtering from the channel with impulse response $c(t)$, the distortion by additive Gaussian noise, $\tilde{n}(t)$, and the demodulation by the imperfect $\mathrm{LO}$ at the receiver is given by

$$
\begin{aligned}
z(t) & =e^{-j \theta_{R}(t)} \int_{-\infty}^{+\infty} c\left(\tau_{1}\right) s\left(t-\tau_{1}\right) \mathrm{d} \tau_{1}+e^{-j \theta_{R}(t)} \tilde{n}(t) \\
& =e^{-j \theta_{R}(t)} \int_{-\infty}^{+\infty} c\left(\tau_{1}\right)\left(e^{j \theta_{T}\left(t-\tau_{1}\right)} \sum_{\nu=1}^{N} x_{\nu} g_{T}\left(t-\tau_{1}-\nu T_{s}\right)\right) \mathrm{d} \tau_{1}+e^{-j \theta_{R}(t)} \tilde{n}(t) \\
& =\sum_{\nu=1}^{N} x_{\nu} \int_{-\infty}^{+\infty} e^{-j\left(\theta_{R}(t)-\theta_{T}\left(t-\tau_{1}\right)\right)} c\left(\tau_{1}\right) g_{T}\left(t-\tau_{1}-\nu T_{s}\right) \mathrm{d} \tau_{1}+n(t)
\end{aligned}
$$


At the output of the receive filter, $g_{R}(t)$, the signal is given by

$$
\begin{aligned}
y(t) & =\int_{-\infty}^{+\infty} g_{R}\left(\tau_{2}\right) z\left(t-\tau_{2}\right) \mathrm{d} \tau_{2} \\
& =\sum_{\nu=1}^{N} x_{\nu} \int_{-\infty}^{+\infty} \int_{-\infty}^{+\infty} e^{-j\left(\theta_{R}\left(t-\tau_{2}\right)-\theta_{T}\left(t-\tau_{2}-\tau_{1}\right)\right)} \\
& \times g_{R}\left(\tau_{2}\right) c\left(\tau_{1}\right) g_{T}\left(t-\tau_{2}-\tau_{1}-\nu T_{s}\right) \mathrm{d} \tau_{1} \mathrm{~d} \tau_{2}+\int_{-\infty}^{+\infty} g_{R}\left(\tau_{2}\right) n\left(t-\tau_{2}\right) \mathrm{d} \tau_{2}
\end{aligned}
$$

It is clear that a discrete-time model derived from the continuous-time representation is necessary. A rigorous argumentation that can lead to a tractable discrete-time description appears to be difficult. Often an approximation similar to the one applied to discretize (3.9) is invoked. That is, the phase noise processes are assumed to be significantly more narrowband than the phase-noise-free overall pulse shape $h(t)=\left(g_{R} * c * g_{T}\right)(t)$. Under this assumption, the transmit and receive phase noise processes are treated as one and the discrete-time model is given by

$$
y_{i}=e^{-j \theta[i]} \sum_{l=0}^{L-1} h_{l} x_{i-l}+n_{i},
$$

where $h_{l}=\left(g_{R} * c * g_{T}\right)\left(l T_{s}\right)^{1}$ and $\theta[i]$ is the sampled equivalent phase noise process. Such an approach has been adopted by many authors, such as [28, 35-37], which are studies of single-input single-output (SISO) orthogonal frequency division multiplexing (OFDM) systems.

There are two outstanding issues with the discrete-time model in (3.13). First, the phase noise processes at the transmitter and the receiver are treated as an equivalent phase noise process. This is a problem in MIMO and multi-user systems, where independent phase noise processes at different antenna elements or at different users must be appear separately. Second, the phase noise processes at the transmitter and the receiver, $\theta_{T}(t)$ and $\theta_{R}(t)$, in (3.12) are distinguishable as $e^{j \theta_{T}(t)}$ is convolved with the propagation channel, $c(t)$, as well as with the receive filter, $g_{R}(t)$. In contrast, $e^{-j \theta_{R}(t)}$ is only convolved with $g_{R}(t)$. We seek for a discrete-time approximation that will reflect this property of the continuous-time representation.

\footnotetext{
${ }^{1}$ Here, it has also been assumed that the overall pulse shape, $h(t)$, gradually decays to negligible values, so we can truncate the sampled impulse response to $L$ symbol-spaced channel taps.
} 
We assume that the transmit and receive filters, $g_{T}(\cdot)$ and $g_{R}(\cdot)$, are ideal integrate-and-dump filters, i.e., their impulse responses are given by

$$
g_{T}(t)=g_{R}(t)= \begin{cases}\frac{1}{\sqrt{T_{s}}}, & 0 \leq t \leq T_{s} \\ 0, & \text { elsewhere }\end{cases}
$$

Then the continuous-time model in (3.12) is expressed as

$$
\begin{aligned}
y(t) & =\sum_{\nu=1}^{N} x_{\nu} \int_{0}^{T_{s}} \int_{t-\tau_{2}-\nu T_{s}-T_{s}}^{t-\tau_{2}-\nu T_{s}} e^{-j\left(\theta_{R}\left(t-\tau_{2}\right)-\theta_{T}\left(t-\tau_{2}-\tau_{1}\right)\right)} \frac{1}{T_{s}} c\left(\tau_{1}\right) \mathrm{d} \tau_{1} \mathrm{~d} \tau_{2} \\
& +\int_{0}^{T_{s}} \frac{1}{\sqrt{T_{s}}} n\left(t-\tau_{2}\right) \mathrm{d} \tau_{2}
\end{aligned}
$$

With equidistant symbol-spaced sampling at $t=i T_{s}$, the discrete-time model derived from the continuous-time model in (3.15) is given by

$$
\begin{aligned}
y_{i} & =y\left(t=i T_{s}\right) \\
& =\sum_{\nu=1}^{N} x_{l} \int_{0}^{T_{s}} \int_{(i-\nu-1) T_{s}-\tau_{2}}^{(i-\nu) T_{s}-\tau_{2}} e^{-j\left(\theta_{R}\left(i T_{s}-\tau_{2}\right)-\theta_{T}\left(i T_{s}-\tau_{2}-\tau_{1}\right)\right)} \frac{1}{T_{s}} c\left(\tau_{1}\right) \mathrm{d} \tau_{1} \mathrm{~d} \tau_{2} \\
& +\int_{0}^{T_{s}} \frac{1}{\sqrt{T_{s}}} n\left(i T_{s}-\tau_{2}\right) \mathrm{d} \tau_{2} .
\end{aligned}
$$

We invoke the piecewise-continuous assumption on the phase noise processes successively on $\theta_{T}(\cdot)$ and $\theta_{R}(\cdot)$, i.e., we assume that the phase noise process has not changed substantially within an interval of duration $T_{s}$, and we also define

$$
\tilde{c}(t) \triangleq \frac{1}{T_{c}} \int_{t-T_{s}}^{t} c(\tau) \mathrm{d} \tau
$$

and

$$
n_{i} \triangleq \int_{0}^{T_{s}} \frac{1}{\sqrt{T_{s}}} n\left(i T_{s}-\tau\right) \mathrm{d} \tau .
$$

Then, the discrete-time approximation is given by

$$
\begin{aligned}
y_{i} & \approx \sum_{\nu=1}^{N} x_{\nu} e^{j \theta_{T}\left(\nu T_{s}\right)} \int_{0}^{T_{s}} e^{-j \theta_{R}\left(i T_{s}-\tau_{2}\right)} \tilde{c}\left((i-\nu) T_{s}-\tau_{2}\right) \mathrm{d} \tau_{2}+n_{i} \\
& \approx \sum_{\nu=1}^{N} x_{\nu} e^{-j \theta_{R}\left((i-1) T_{s}\right)} e^{j \theta_{T}\left(\nu T_{s}\right)} \int_{0}^{T_{s}} \tilde{c}\left((i-\nu) T_{s}-\tau_{2}\right) \mathrm{d} \tau_{2}+n_{i} .
\end{aligned}
$$


With the additional definition and truncation of the discrete-time impulse response

$$
h_{l} \triangleq \begin{cases}\int_{0}^{T_{s}} \tilde{c}\left(l T_{s}-\tau\right) \mathrm{d} \tau, & \text { for } 0 \leq l \leq L-1 \\ 0, & \text { elsewhere }\end{cases}
$$

a discrete-time approximation of the continuous-time model in (3.12) is given by

$$
y_{i}=e^{-j \theta_{R}[i]} \sum_{l=0}^{L-1} h_{l} e^{j \theta_{T}[i-l]} x_{i-l}+n_{i} .
$$

The system model in (3.20) is appealing since the phase noise processes at the transmitter and the receiver appear explicitly and the convolution effect of the propagation channel on $\theta_{T}(t)$ is also given as a discrete-time convolutional sum. Consequently, it has been extensively used in the literature to model phase noise in frequency selective channels [38-40]. However, in contrast to the discrete-time model in (3.10), a detailed derivation of the model (3.20), as the one given here, appears to be absent in the relevant literature.

\subsection{The Wiener Phase Noise Model in Communica- tions}

In the Sections 3.1, 3.2 and 3.3 we described the way that phase noise appears in standard communication system models [38,41-44]. In this section we present a widely studied model on phase noise in the fields of Information Theory and Detection \& Estimation Theory. Based on the discussion of Section 2.3.3, the phase noise process, $\theta(t)=\omega_{c} \alpha(t)$, in (3.8) is modeled as a continuous-time Wiener process, i.e.,

$$
\theta(t)-\theta\left(t_{0}\right)=\int_{t_{0}}^{t} w(\tau) \mathrm{d} \tau,
$$

where $t_{0}$ is the start of the observation of the process and $w(t)$ is a real white Gaussian process with $\mathbb{E}[w(t)]=0$ and $\mathbb{E}\left[w\left(t_{1}\right) w\left(t_{2}\right)\right]=\omega_{c}^{2} c \delta\left(t_{1}-t_{2}\right)$. Of particular interest is the autocorrelation and power spectral density of the 
process $\zeta(t)=e^{j\left(\theta(t)-\theta\left(t_{0}\right)\right)}$. It is straightforward from the definitions of $\zeta(t)$ and $\theta(t)$ to show that

$$
\begin{aligned}
R_{\zeta}(\tau) & =\mathbb{E}\left[\zeta(t) \zeta^{*}(t+\tau)\right]=\mathbb{E}\left[e^{j\left(\theta(t)-\theta\left(t_{0}\right)\right)} e^{-j\left(\theta(t+\tau)-\theta\left(t_{0}\right)\right)}\right] \\
& =\mathbb{E}\left[e^{j \omega_{c}(\alpha(t)-\alpha(t+\tau))}\right]=e^{-\frac{\omega_{c}^{2} c}{2}|\tau|}
\end{aligned}
$$

The fact that $R_{\zeta}(\tau)$ is only function of the relative time lag $\tau$ implies the stationarity of the process $\zeta(t)$. The corresponding power spectral density is

$$
\begin{aligned}
S_{\zeta}(f) & =\int_{-\infty}^{+\infty} R_{\zeta}(\tau) e^{-j 2 \pi f \tau} \mathrm{d} \tau=\int_{-\infty}^{+\infty} e^{-\frac{\omega_{c}^{2} c}{2}|\tau|} e^{-j 2 \pi f \tau} \mathrm{d} \tau \\
& =\frac{1}{\pi} \frac{\pi f_{c}^{2} c}{\pi^{2} f_{c}^{4} c^{2}+f^{2}}=\frac{1}{\pi} \frac{\beta / 2}{(\beta / 2)^{2}+f^{2}} .
\end{aligned}
$$

The shape of the spectrum in (3.23) is Lorentzian and $\beta \triangleq 2 \pi f_{c}^{2} c$ is the double-sided $3 \mathrm{~dB}$ bandwidth of the process. Observe that this shape is an accurate description of the medium offset region of $\mathcal{L}(\Delta \omega)$ in Fig. 2.5. However, it does not exhibit the $1 / f^{3}$ behavior for small offsets from carrier. This is due to the fact that the Wiener phase noise characterization is valid for LOs with only white circuit noise sources (thermal and shot noise) and the $1 / f^{3}$ behavior is attributed to colored circuit noise sources, e.g., flicker noise.

In the discrete time, the continuous-time Wiener process is sampled at a frequency $1 / T_{s}$ to yield the discrete-time Wiener process, where

$$
\theta_{i+1}=\theta_{i}+w_{i}
$$

where $\theta_{i}=\theta\left(i T_{s}\right)$ and

$$
w_{i}=\int_{i T_{s}}^{(i+1) T_{s}} w(\tau) \mathrm{d} \tau .
$$

From the properties of the continuous-time Wiener process, the increments $w_{i}$ are independent identically distributed (i.i.d.) zero mean real Gaussian random variables with variance $\sigma_{w}^{2}=2 \pi \beta T_{s}$, i.e., $w_{i} \sim \mathcal{N}_{\mathbb{R}}\left(0, \sigma_{w}^{2}\right)$. In information-theoretic studies it is often assumed that the initial phase $\theta_{0}$ is uniform in $[0,2 \pi)$, i.e., $\theta_{0} \sim \mathcal{U}[0,2 \pi)$, and then the process $\left\{\theta_{i}\right\}$ is stationary [45]. 


\section{Chapter 4}

\section{Massive MIMO with Phase Noise Impairments}

In this chapter, the concept of MU-MIMO systems is introduced and the basic system model is described for the single-cell case. The introduction of Massive MIMO follows, where the main differences with conventional MU-MIMO systems are explained. The outline of the achievable sum-rate analysis in Massive MIMO is briefly presented. Subsequently, phase noise impairments are introduced into the basic Massive MIMO model. Through simple yet illustrative examples, the fundamental differences in the achievable sum-rate analysis between the phase noise case and phase-noise-free case are reviewed. The capacity lower bounds used in this dissertation are presented in detail, their desirable characteristics are mentioned and the potential use of alternative capacity lower bounds is discussed.

\subsection{The MU-MIMO Uplink Channel}

Consider a BS with $M$ antenna elements serving simultaneously $K$ singleantenna, non-cooperative users ${ }^{1}$ within a geographical area, called cell. We study the direction of communication where the users send data to the BS; this is called uplink. Narrowband transmission is assumed, i.e., the effect

\footnotetext{
${ }^{1}$ The single-antenna per user assumption is not essential and is done here for simplicity. However, the non-cooperation between users is fundamental.
} 
of the propagation through the channel between a user and a BS antenna is represented by a single complex scalar coefficient. We denote the channel gain from the $k$-th user to the $m$-th BS antenna by $h_{m, k}$ and the channel vector from the $k$-th user to the BS by $\boldsymbol{h}_{k} \triangleq\left[h_{1, k}, \ldots, h_{M, k}\right]^{T}$ and assume that it is a circularly symmetric complex Gaussian random vector, i.e., $\boldsymbol{h}_{k} \sim$ $\mathcal{N}_{\mathbb{C}}\left(\mathbf{0}, \boldsymbol{I}_{M}\right)$. If the $k$-th user transmits the symbol $x_{k}$, which is subject to an average power constraint, $\mathbb{E}\left[\left|x_{k}\right|^{2}\right] \leq 1$, then the received vector at the BS is given by

$$
\boldsymbol{y}=\sqrt{\rho} \sum_{k=1}^{K} \boldsymbol{h}_{k} x_{k}+\boldsymbol{w}_{k},
$$

where $\boldsymbol{y} \in \mathbb{C}^{M}$ is an $M$-dimensional complex vector and $\boldsymbol{w}_{k}$ is additive white Gaussian noise, distributed as $\boldsymbol{w}_{k} \sim \mathcal{N}_{\mathbb{C}}\left(\mathbf{0}, \boldsymbol{I}_{M}\right)$. If $\mathbb{E}\left[\left|h_{m, k}\right|^{2}\right]=$ $1, \forall m, k$ and only one user transmits, then $\rho$ is the expected signal-to-noiseratio (SNR) at each BS antenna. Important research results on the limits of communication on the uplink channel are already available in the literature [46-49]. The downlink, which is the communication direction from the BS to the users, is also important and has been extensively studied [50-57].

The concept of MU-MIMO systems was in itself a paradigm shift with respect to point-to-point single-user (SU) MIMO. In point-to-point SU-MIMO both the BS and the user terminal are equipped with multiple antenna elements and different users are scheduled in orthogonal channels. In the presence of a strong LoS path between the user and the BS or of strong spatial correlation, the number of streams that can be multiplexed in the SU-MIMO case can drop to one, offering only a small performance gain in comparison to the case where the BS and the user have a single antenna. However, MU-MIMO is more robust under LoS conditions, since two users are likely to be spatially separated and cases of strong correlation among users can be resolved by appropriate multi-user scheduling. Also, due to the singleantenna assumption for the user terminals, the complexity is moved to the BS and the user terminals can be small and energy efficient. However, MUMIMO has also drawbacks. Accurate knowledge of the channels, $\boldsymbol{h}_{k}$, is required to reap the gains of MU-MIMO. This knowledge acquisition is costly, particularly in the downlink direction [5]. In addition, the optimal transmit and receive strategies in MU-MIMO, where the number of BS antennas, $M$, is approximately equal to the number of single-antenna users, $K$, are nonlinear with very high complexity and simpler linear schemes often perform poorly. 


\subsection{Massive MIMO}

Massive MIMO proposes a further shift in the paradigm of cellular systems design $[58,59]$. A Massive MIMO BS is equipped with an unprecedentedly large number of antenna elements, $M$, a few hundreds or even thousands, and serves simultaneously a few tens of non-cooperative, single-antenna users, $K$. In this operational regime, where $M \gg K$, the normalized propagation vector channels from different users tend to become asymptotically orthogonal. This is called favorable propagation. Further, the normalized channel norms tend to be very close to their statistical mean. This phenomenon is called channel hardening. With favorable propagation and channel hardening, linear transmit and receive strategies are close to optimal. Similar to conventional MU-MIMO, accurate knowledge of the channels at the BS side is required in Massive MIMO in order to reap the promised gains. This knowledge is typically acquired via uplink training. With this choice, the length of the required training interval increases with the number of terminals and not with the number of BS antennas. Finally, the BS can use the channel knowledge acquired via uplink training to detect the signals received in the uplink.

The concept of coherence interval is key in the study of wireless channels, and of Massive MIMO in particular. A coherence interval is the block, of say $\tau_{c}$ channel uses, during which the channel remains approximately constant. In the present exposition of Massive MIMO the coherence interval is split into an uplink training interval of $\tau_{p} \geq K$ channel uses and an uplink data interval of $\tau_{d}=\tau_{c}-\tau_{p}$ channel uses ${ }^{2}$. During training, each user transmits a deterministic training sequence $\sqrt{\tau_{p}} \psi_{k}$ that is orthogonal to the training sequences of the other users, i.e., $\boldsymbol{\psi}_{k}^{H} \boldsymbol{\psi}_{k^{\prime}}=\delta\left(k-k^{\prime}\right)$, where $\delta(\cdot)$ is the Kronecker delta. The received vectors during training are given by

$$
\begin{aligned}
\boldsymbol{Y}_{p} & =\sqrt{\rho \tau_{p}} \sum_{k=1}^{K} \boldsymbol{h}_{k} \boldsymbol{\psi}_{k}^{T}+\boldsymbol{W}_{p} \\
\boldsymbol{Y}_{p} \boldsymbol{\psi}_{k}^{*} & =\sqrt{\rho \tau_{p}} \boldsymbol{h}_{k}+\boldsymbol{W}_{p} \boldsymbol{\psi}_{k}^{*},
\end{aligned}
$$

where $\boldsymbol{Y}_{p}=\left[\boldsymbol{y}[1], \ldots, \boldsymbol{y}\left[\tau_{p}\right]\right]$ and $\boldsymbol{W}_{p}=\left[\boldsymbol{w}[1], \ldots, \boldsymbol{w}\left[\tau_{p}\right]\right]$. The BS forms an estimate, $\hat{\boldsymbol{h}}_{k}$, for the channel vector, $\boldsymbol{h}_{k}$, based on $\boldsymbol{Y}_{p} \boldsymbol{\psi}_{k}^{*}$ in order to detect the

\footnotetext{
${ }^{2}$ In Massive MIMO part of the coherence interval is allocated also for downlink data transmission and, possibly, for downlink pilots [60], but in this introductory exposition the focus is on the uplink.
} 
information symbols transmitted from the $k$-th user, $x_{k}\left[\tau_{p}+1\right], \ldots, x_{k}\left[\tau_{c}\right]$. Information symbols from different users are assumed to be independent. During the data transmission interval, the BS uses the estimated channels ${ }^{3}$ with maximum ratio combining (MRC) ${ }^{4}$ to detect the transmitted data symbol, $x_{k}[i]$, at the $i$-th channel use of the data interval, i.e.,

$$
\hat{x}_{k}[i]=\hat{\boldsymbol{h}}_{k}^{H} \boldsymbol{y}[i]=\sqrt{\rho} \sum_{k^{\prime}=1}^{K} \hat{\boldsymbol{h}}_{k}^{H} \boldsymbol{h}_{k^{\prime}} x_{k^{\prime}}[i]+\hat{\boldsymbol{h}}_{k}^{H} \boldsymbol{w}[i] .
$$

If $\mathbb{E}\left[x_{k}^{*} \hat{\boldsymbol{h}}_{k}^{H} \boldsymbol{w}[i] \mid \hat{\boldsymbol{h}}_{k}\right]=0$ and $\mathbb{E}\left[\left(\hat{\boldsymbol{h}}_{k}^{H} \boldsymbol{h}_{k} x_{k}\right)^{*} \hat{\boldsymbol{h}}_{k}^{H} \boldsymbol{w}[i] \mid \hat{\boldsymbol{h}}_{k}\right]=0$, a lower bound (achievable rate), $R_{k}^{S I}$, on the maximum mutual information between $x_{k}[i]$ and $\hat{x}_{k}[i]$ conditioned on $\hat{\boldsymbol{h}}_{k}, I\left(x_{k}[i] ; \hat{x}_{k}[i] \mid \hat{\boldsymbol{h}}_{k}\right)$ is obtained by

$$
\begin{aligned}
& \max _{p_{X_{k}[i]}\left(x_{k}[i]\right)} I\left(x_{k}[i] ; \hat{x}_{k}[i] \mid \hat{\boldsymbol{h}}_{k}\right) \geq R_{k}^{\mathrm{SI}} \\
& =\mathbb{E}\left[\log _{2}\left(1+\frac{\rho\left|\mathbb{E}\left[\hat{\boldsymbol{h}}_{k}^{H} \boldsymbol{h}_{k} \mid \hat{\boldsymbol{h}}_{k}\right]\right|^{2}}{\rho \sum_{k^{\prime}=1}^{K} \mathbb{E}\left[\left|\hat{\boldsymbol{h}}_{k}^{H} \boldsymbol{h}_{k^{\prime}}\right|^{2} \mid \hat{\boldsymbol{h}}_{k}\right]-\rho\left|\mathbb{E}\left[\hat{\boldsymbol{h}}_{k}^{H} \boldsymbol{h}_{k} \mid \hat{\boldsymbol{h}}_{k}\right]\right|^{2}+\left\|\hat{\boldsymbol{h}}_{k}\right\|^{2}}\right)\right] .
\end{aligned}
$$

The maximization is over all the input densities $p_{X_{k}[i]}\left(x_{k}[i]\right)$ that satisfy the power constraint $\mathbb{E}\left[\left|x_{k}[i]\right|^{2}\right] \leq 1$. We will use the term side-information bound for $R^{\text {SI }}$ since it explicitly uses the side information acquired via uplink training both to process the received signal vector $\boldsymbol{y}[i]$ and to decode the information symbol $x_{k}[i]$ from the output, $\hat{x}_{k}[i]$, of the receive processing filter. A more tractable but looser bound, $R_{k}^{\mathrm{UNF}}$, on the maximum mutual information between $x_{k}[i]$ and $\hat{x}_{k}[i], I\left(x_{k}[i] ; \hat{x}_{k}[i]\right)$, is given by

$$
\begin{aligned}
& \max _{p_{X_{k}[i]}\left(x_{k}[i]\right)} I\left(x_{k}[i] ; \hat{x}_{k}[i]\right) \geq R_{k}^{\mathrm{UNF}} \\
& =\log _{2}\left(1+\frac{\rho\left|\mathbb{E}\left[\hat{\boldsymbol{h}}_{k}^{H} \boldsymbol{h}_{k}\right]\right|^{2}}{\rho \sum_{k^{\prime}=1}^{K} \mathbb{E}\left[\left|\hat{\boldsymbol{h}}_{k}^{H} \boldsymbol{h}_{k^{\prime}}\right|^{2}\right]-\rho\left|\mathbb{E}\left[\hat{\boldsymbol{h}}_{k}^{H} \boldsymbol{h}_{k}\right]\right|^{2}+\mathbb{E}\left[\left\|\hat{\boldsymbol{h}}_{k}\right\|^{2}\right]}\right) .
\end{aligned}
$$

We will use the term use-and-forget bound for $R^{\mathrm{UNF}}$ since $\hat{\boldsymbol{h}}_{k}$ is only used to process the received vector $\boldsymbol{y}[i]$ but this information is not explicitly used to

\footnotetext{
${ }^{3}$ The users have no knowledge of the estimates $\hat{\boldsymbol{h}}_{k}$, hence the data symbols $x_{k}[i]$ are independent of the estimates $\hat{\boldsymbol{h}}_{k}$.

${ }^{4}$ Some other linear or with low complexity processing scheme, such as linear minimum mean square error (LMMSE), is also possible.
} 
decode the information symbol $x_{k}[i]$ from $\hat{x}_{k}[i]$. A detailed derivation and information-theoretic justification of these bounds can be found in [61]. We note that these bounds first appeared in [62-64] and since then they have been extensively used in the study of Massive MIMO systems [10,11,65-67].

\subsection{Massive MIMO with Phase Noise}

In this section the basic single-cell system model in (4.1) is augmented with the effect of transmit and receive phase noise and the methodology for the derivation of achievable rates is outlined and explained. For simplicity, the exposition here is restricted to a single user, however, all the fundamental phenomena that appear due to phase noise are readily revealed. The treatment of more complicated models can be found in the papers that follow. At the $i$-th channel use of the coherence interval, the received signal at the $m$-th BS antenna is given by

$$
y_{m}[i]=\sqrt{\rho} e^{-j \theta_{m}[i]} h_{m} e^{j \phi[i]} x[i]+w_{m}[i],
$$

based on the analysis leading to (3.20) (with $L=1$ ). The processes $\theta_{m}[\cdot]$, $\phi[\cdot], h_{m}, x[\cdot]$ and $w_{m}[\cdot]$ are mutually independent. However, BS phase noise processes from the $m_{1}$-th and $m_{2}$-th BS antennas, $\theta_{m_{1}}[\cdot]$ and $\theta_{m_{2}}[\cdot]$, respectively, can be arbitrarily dependent. We consider two particular operations; the synchronous operation where $\theta_{1}[\cdot] \equiv \cdots \equiv \theta_{M}[\cdot]$ and the non-synchronous operation where the $\theta_{m}[\cdot]$ are mutually independent along the BS antennas. The synchronous operation models a centralized deployment where one $\mathrm{LO}$ provides the carrier waveform to all the BS antennas. In contrast, the non-synchronous operation models a distributed deployment where separate LOs are used for every BS antenna. All the phase noise processes are assumed to be discrete-time Wiener processes as defined in Section 3.4. The variances of the phase noise increments for $\theta_{m}[\cdot]$ and $\phi[\cdot]$ are $\sigma_{\theta}^{2}$ and $\sigma_{\phi}^{2}$, respectively. The matrix-vector formulation is given by

$$
\boldsymbol{y}[i]=\sqrt{\rho} \boldsymbol{\Theta}[i] \boldsymbol{h} e^{j \phi[i]} x[i]+\boldsymbol{w}[i],
$$

where $\boldsymbol{y}[i] \in \mathbb{C}^{M}, \boldsymbol{\Theta}[i] \triangleq \operatorname{diag}\left\{e^{-j \theta_{1}[i]}, \ldots, e^{-j \theta_{M}[i]}\right\}$ for the nonsynchronous operation and $\Theta[i] \triangleq e^{-j \theta[i]} \boldsymbol{I}_{M}$ for the synchronous operation.

As it has been outlined in Section 4.2, a coherence interval of $\tau_{c}$ channel uses is assumed with $\tau_{p}$ channel uses for uplink training and $\tau_{d}$ channel 
uses for uplink data transmission. The propagation channel, $\boldsymbol{h}$, remains constant for the coherence interval, changes to an independent realization between different coherence intervals and the fading process is assumed to be stationary and ergodic.

\subsubsection{Uplink Training}

For simplicity, the training sequence consists of $\tau_{p}$ ones. At the $l$-th channel use of the training interval the received signal, $\boldsymbol{y}[l]$, is given by

$$
\boldsymbol{y}[l]=\sqrt{\rho} \boldsymbol{\Theta}[l] \boldsymbol{h} e^{j \phi[l]}+\boldsymbol{w}[l] .
$$

It is clear that, even though $h$ remains constant during the coherence interval, the phase noise processes evolve at symbol rate. Hence, $\boldsymbol{h}$ has to be estimated in the presence of the time-varying disturbance due to phase noise. We select to use the following estimate

$$
\hat{\boldsymbol{h}}=\frac{1}{\tau_{p} \sqrt{\rho}} \sum_{l=1}^{\tau_{p}} \boldsymbol{y}[l]=\frac{1}{\tau_{p}} \sum_{l=1}^{\tau_{p}} \boldsymbol{\Theta}[l] \boldsymbol{h} e^{j \phi[l]}+\frac{1}{\tau_{p} \sqrt{\rho}} \sum_{l=1}^{\tau_{p}} \boldsymbol{w}[l] .
$$

This particular estimate is the ML estimate of $\boldsymbol{h}$ given the received vectors $\boldsymbol{y}[1], \ldots, \boldsymbol{y}\left[\tau_{p}\right]$ in the absence of phase noise and when $\boldsymbol{w}[l]$ is Gaussian. It is also linear and with low complexity, which are important requirements for channel estimation in Massive MIMO. The use of the minimum mean square error (MMSE) estimate is another choice. Various fundamental papers on Massive MIMO [9-11] have used this estimate. In (4.9) the coefficients of the effective vector channels are rotations of the propagation channel, $\boldsymbol{h}$, due to the presence of phase noise. Hence, the calculation of the MMSE estimate is formidable. The calculation of the linear MMSE estimate is straightforward, however, its use does not make the derivation of the achievable rates substantially easier with respect to the "quasi"-ML estimate of (4.10) nor does it change the fundamental insights drawn from the final result.

\subsubsection{Data Transmission}

The BS uses the channel knowledge acquired via training in (4.10) to perform MRC on the received vectors, $\boldsymbol{y}[i]$, during data transmission. The detected symbol, $\hat{x}[i]$, based on processing of the received vector $\boldsymbol{y}[i]$ is given 
by

$$
\hat{x}[i]=\hat{\boldsymbol{h}}^{H} \boldsymbol{y}[i]=\sqrt{\rho} \hat{\boldsymbol{h}}^{H} \boldsymbol{\Theta}[i] \boldsymbol{h} e^{j \phi[i]} x[i]+\hat{\boldsymbol{h}}^{H} \boldsymbol{w}[i] .
$$

Observe that even though $\hat{\boldsymbol{h}}$ provides important information about $\boldsymbol{h}$, this is in the form of a superposition of various versions of $\boldsymbol{h}$ with elements that are randomly rotated by phase noise. Further, the effective channel gains at the $i$-th channel use, $\Theta[i] \boldsymbol{h} e^{j \phi[i]}$, are also rotated versions of the propagation channel, $\boldsymbol{h}$. Therefore, it appears formidable to exploit all the information available by the channel estimate, $\hat{\boldsymbol{h}}$, further than its use in (4.11), as it was done in (4.5). An extension of the approach leading to (4.6) is henceforth outlined. By expanding the estimated channel, $\hat{\boldsymbol{h}}$, the detected symbol, $\hat{x}[i]$, in (4.11) is written as

$$
\hat{x}[i]=\sqrt{\rho} A[i] x[i]+\frac{1}{\tau_{p}} \sum_{l=1}^{\tau_{p}} \boldsymbol{w}^{H}[l] \Theta[i] \boldsymbol{h} e^{j \phi[i]} x[i]+\hat{\boldsymbol{h}}^{H} \boldsymbol{w}[i],
$$

where

$$
A[i] \triangleq \frac{1}{\tau_{p}} \sum_{l=1}^{\tau_{p}} \boldsymbol{h}^{H} \boldsymbol{\Theta}^{H}[l] \boldsymbol{\Theta}[i] \boldsymbol{h} e^{j(\phi[i]-\phi[l])} .
$$

Observe that $A[i]$ is random due to its dependence on phase noise. However, its moments can be computed. Hence, we can express (4.12) as

$$
\hat{x}[i]=\sqrt{\rho \mathbb{E}}[A[i]] x[i]+\mathrm{EN}[i]
$$

where the effective noise term $\operatorname{EN}[i]$ is given by

$$
\operatorname{EN}[i]=\sqrt{\rho}(A[i]-\mathbb{E}[A[i]]) x[i]+\frac{1}{\tau_{p}} \sum_{l=1}^{\tau_{p}} \boldsymbol{w}^{H}[l] \Theta[i] \boldsymbol{h} e^{j \phi[i]} x[i]+\hat{\boldsymbol{h}}^{H} \boldsymbol{w}[i] .
$$

Observe that $\mathbb{E}[A[i]] x[i]$ and $\operatorname{EN}[i]$ are uncorrelated but they are not necessarily independent. With $x_{k}[i] \sim \mathcal{N}_{\mathbb{C}}(0,1)$ the worst case additive uncorrelated noise, with respect to mutual information, is circularly symmetric complex Gaussian with 0 mean and variance equal to the variance of $\operatorname{EN}[i]$. Hence, a lower bound on the mutual information $I(x[i] ; \hat{x}[i])$ is given by

$$
I(x[i] ; \hat{x}[i]) \geq R^{\mathrm{UNF}}[i]=\log _{2}\left(1+\frac{\rho|\mathbb{E}[A[i]]|^{2}}{\operatorname{VAR}(\operatorname{EN}[i])}\right),
$$


where

$$
\mathbb{E}[A[i]]=\frac{M}{\tau_{p}} \sum_{l=1}^{\tau_{p}} e^{-\frac{\sigma_{\phi}^{2}+\sigma_{\theta}^{2}}{2}|i-l|} .
$$

For the particular cases of synchronous and non-synchronous operation the expression $\operatorname{VAR}(\operatorname{EN}[i])$ is

$$
\operatorname{VAR}(\operatorname{EN}[i])=\rho\left(M(M+1) t_{1}-|\mathbb{E}[A[i]]|^{2}\right)+M t_{1}+\frac{M}{\tau_{p} \rho}+\frac{M}{\tau_{p}},
$$

for the synchronous operation and

$$
\operatorname{VAR}(\operatorname{EN}[i])=\rho\left(2 M t_{1}+M(M-1) t_{2}-|\mathbb{E}[A[i]]|^{2}\right)+M t_{1}+\frac{M}{\tau_{p} \rho}+\frac{M}{\tau_{p}},
$$

for the non-synchronous operation, where the terms $t_{1}$ and $t_{2}$ are given by

$$
\begin{aligned}
& t_{1}=\frac{1}{\tau_{p}^{2}} \sum_{l_{1}=1}^{\tau_{p}} \sum_{l_{2}=1}^{\tau_{p}} e^{-\frac{\sigma_{\phi}^{2}+\sigma_{\theta}^{2}}{2}\left|l_{1}-l_{2}\right|} \\
& t_{2}=\frac{1}{\tau_{p}^{2}} \sum_{l_{1}=1}^{\tau_{p}} \sum_{l_{2}=1}^{\tau_{p}} e^{-\frac{\sigma_{\phi}^{2}}{2}\left|l_{1}-l_{2}\right|} e^{-\frac{\sigma_{\theta}^{2}}{2}\left|i-l_{1}\right|} e^{-\frac{\sigma_{\theta}^{2}}{2}\left|i-l_{2}\right|} .
\end{aligned}
$$

The overall effective achievable rate is given then by

$$
R^{\mathrm{UNF}}=\frac{1}{\tau_{c}} \sum_{i=\tau_{p}+1}^{\tau_{c}} R^{\mathrm{UNF}}[i] .
$$

The result in (4.22) has certain appealing properties. First, it is in closedform. This implies that no time-consuming and complicated numerical studies are required to predict the performance of phase-noise-impaired Massive MIMO. The effects on the achievable rate with respect to parameters, such as the number of the BS antennas, $M$, the variance of the phase noise increments, $\sigma_{\phi}^{2}$ and $\sigma_{\theta}^{2}$ and the length of the training interval, $\tau_{p}$, appear explicitly in the derived achievable rate. This facilitates the statement of various scaling laws with respect to the individual parameters, e.g., how does the achievable rate change as a function of $M$. Also, connections between different parameters can be revealed, e.g., how much can $\rho$ be reduced with an increase of $M$, while a fixed desired achievable rate is guaranteed. 
The achievable rate in (4.22) corresponds to a realistic scenario, i.e., an explicit and implementable channel estimation scheme is used to acquire CSI, the acquired CSI is used for data detection with low complexity, linear reception and the effect of the finite coherence interval is made explicit. The methodology provides insights for different deployment scenarios. Here, the synchronous and non-synchronous operations have been considered but similar analysis can be performed for arbitrary hybrid scenarios, where a single LO controls a group of antenna elements. Further, there is a plethora of results for phase-noise-free Massive MIMO, which are derived based on similar methodology [10,66,68], as outlined in Section 4.2. Quantifying the achievable rate loss due to phase noise in Massive MIMO is now straightforward by comparison to the existing results. Finally, the methodology applied here can be extended to more complex scenarios, e.g., multiple users, multiple cells, and also to more complex system models that include other hardware impairments $[69,70]$.

\subsection{Alternative Capacity Bound for Massive MIMO with Phase Noise}

In the previous section the main capacity lower bound used in the dissertation was explained and justified. In this section we explore a special case where another achievable rate can be derived, based on the analysis leading to (4.5). That is, in this bound the acquired channel knowledge is exploited both for MRC detection and for symbol decoding. Even though, the approach seems difficult to be extended to realistic single-cell Massive MIMO systems with phase noise, its derivation and comparison with the used bound provides important insights on the gap in terms of achievable rate given by (4.16) and (4.22). We note that for the derivation of (4.16) and (4.22), part of the information provided by the estimated channel was not used in the decoding process.

Consider the same setup as in Section 4.3 with $\tau_{p}=1$. In this case, the received signal during channel training is given by

$$
\boldsymbol{y}[0]=\sqrt{\rho} \boldsymbol{h}+\boldsymbol{w}[0]
$$

where the initial phase noise rotations have been absorbed into the propagation channel $\boldsymbol{h}$. This is possible without change in the statistics of $\boldsymbol{h}$ 
due to the circular symmetry of the distribution of $\boldsymbol{h}$. The BS calculates the MMSE estimate based on the observation $\boldsymbol{y}[0]$, i.e.,

$$
\hat{\boldsymbol{h}}=\frac{\sqrt{\rho}}{1+\rho} \boldsymbol{y}[0]
$$

Due to the fact that the channel estimate, $\hat{\boldsymbol{h}}$, and the channel estimation error, $\tilde{h}$, are jointly Gaussian and uncorrelated, they are also statistically independent. During data transmission, with MRC processing the detected symbol $\hat{x}[i]$ at the $i$-th channel use is given by

$$
\begin{aligned}
& \hat{x}[i]=\hat{\boldsymbol{h}}^{H} \boldsymbol{y}[i]=\sqrt{\rho} \hat{\boldsymbol{h}}^{H} \boldsymbol{\Theta}[i] \boldsymbol{h} e^{j \phi[i]} x[i]+\hat{\boldsymbol{h}}^{H} \boldsymbol{w}[i] \\
& =\sqrt{\rho \mathbb{E}}\left[\hat{\boldsymbol{h}}^{H} \boldsymbol{\Theta}[i] \boldsymbol{h} e^{j \phi[i]} \mid \hat{\boldsymbol{h}}\right] x[i] \\
& \underbrace{+\sqrt{\rho}\left(\hat{\boldsymbol{h}}^{H} \boldsymbol{\Theta}[i] \boldsymbol{h} e^{j \phi[i]}-\mathbb{E}\left[\hat{\boldsymbol{h}}^{H} \boldsymbol{\Theta}[i] \boldsymbol{h} e^{j \phi[i]} \mid \hat{\boldsymbol{h}}\right]\right) x[i]+\hat{\boldsymbol{h}}^{H} \boldsymbol{w}[i]}_{\triangleq \mathbb{E N}[i]}
\end{aligned}
$$

An ergodic achievable rate is given by

$$
R^{\mathrm{SI}}[i]=\mathbb{E}\left[\log _{2}\left(1+\frac{\rho\left|\mathbb{E}\left[\hat{\boldsymbol{h}}^{H} \boldsymbol{\Theta}[i] \boldsymbol{h} e^{j \phi[i]} \mid \hat{\boldsymbol{h}}\right]\right|^{2}}{\rho \operatorname{VAR}\left(\hat{\boldsymbol{h}}^{H} \Theta[i] \boldsymbol{h} e^{j \phi[i]} \mid \hat{\boldsymbol{h}}\right)+\mathbb{E}\left[\left|\hat{\boldsymbol{h}}^{H} \boldsymbol{w}[i]\right|^{2} \mid \hat{\boldsymbol{h}}\right]}\right)\right] .
$$

The above expression can be further simplified with the calculation of the conditional expectations $\mathbb{E}\left[\hat{\boldsymbol{h}}^{H} \boldsymbol{\Theta}[i] \boldsymbol{h} e^{j \phi[i]} \mid \hat{\boldsymbol{h}}\right]=e^{-\frac{\sigma_{\phi}^{2}+\sigma_{\theta}^{2}}{2} i}\|\hat{\boldsymbol{h}}\|^{2}$ and $\mathbb{E}\left[\hat{\boldsymbol{h}}^{H} \boldsymbol{w}[i] \mid \hat{\boldsymbol{h}}\right]=\|\hat{\boldsymbol{h}}\|^{2}$. The inner expectations are conditioned on $\hat{\boldsymbol{h}}$ and with respect to all the remaining sources of randomness and the outer expectation is with respect to $\hat{\boldsymbol{h}}$. The expression $\operatorname{VAR}\left(\hat{\boldsymbol{h}}^{H} \boldsymbol{\Theta}[i] \boldsymbol{h} e^{j \phi[i]} \mid \hat{\boldsymbol{h}}\right)$ evaluates to

$$
\operatorname{VAR}\left(\hat{\boldsymbol{h}}^{H} \boldsymbol{\Theta}[i] \boldsymbol{h} e^{j \phi[i]} \mid \hat{\boldsymbol{h}}\right)=\left(1-e^{-\left(\sigma_{\phi}^{2}+\sigma_{\theta}^{2}\right) i}\right)\|\hat{\boldsymbol{h}}\|^{4}+\frac{1}{1+\rho}\|\hat{\boldsymbol{h}}\|^{2}
$$

and

$$
\begin{aligned}
\operatorname{VAR}\left(\hat{\boldsymbol{h}}^{H} \boldsymbol{\Theta}[i] \boldsymbol{h} e^{j \phi[i]} \mid \hat{\boldsymbol{h}}\right) & =\left(1-e^{-\sigma_{\theta}^{2} i}\right) \sum_{m=1}^{M}\left|\hat{h}_{m}\right|^{4}+\frac{1}{1+\rho}\|\hat{\boldsymbol{h}}\|^{2} \\
& +e^{-\sigma_{\theta}^{2} i}\left(1-e^{-\sigma_{\phi}^{2} i}\right)\|\hat{\boldsymbol{h}}\|^{4}
\end{aligned}
$$


for the synchronous and non-synchronous operation, respectively. This is a side-information bound that extends the result in (4.5) to Massive MIMO with phase noise.

The corresponding use-and-forget bound can also be derived as outlined in the Section 4.3 and in this case is given by

$$
R^{\mathrm{UNF}}[i]=\log _{2}\left(1+\frac{\rho\left|\mathbb{E}\left[\hat{\boldsymbol{h}}^{H} \boldsymbol{\Theta}[i] \boldsymbol{h} e^{j \phi[i]}\right]\right|^{2}}{\rho \operatorname{VAR}\left(\hat{\boldsymbol{h}}^{H} \boldsymbol{\Theta}[i] \boldsymbol{h} e^{j \phi[i]}\right)+\mathbb{E}\left[\left|\hat{\boldsymbol{h}}^{H} \boldsymbol{w}[i]\right|^{2}\right]}\right),
$$

where the evaluation of the expectations yields $\mathbb{E}\left[\hat{\boldsymbol{h}}^{H} \boldsymbol{\Theta}[i] \boldsymbol{h} e^{j \phi[i]}\right]=$ $\frac{\rho}{\rho+1} M e^{-\frac{\sigma_{\phi}^{2}+\sigma_{\theta}^{2}}{2} i}$ and $\mathbb{E}\left[\left|\hat{\boldsymbol{h}}^{H} \boldsymbol{w}[i]\right|^{2}\right]=\frac{\rho}{\rho+1} M$. The variance term is evaluated for the synchronous operation to

$$
\rho \operatorname{VAR}\left(\hat{\boldsymbol{h}}^{H} \boldsymbol{\Theta}[i] \boldsymbol{h} e^{j \phi[i]}\right)=\rho\left(\frac{\rho}{1+\rho}\right)^{2} M^{2}\left(1-e^{-\left(\sigma_{\phi}^{2}+\sigma_{\theta}^{2}\right) i}\right)+\frac{\rho^{2}}{\rho+1} M
$$

and for the non-synchronous operation to

$$
\begin{aligned}
\rho \operatorname{VAR}\left(\hat{\boldsymbol{h}}^{H} \boldsymbol{\Theta}[i] \boldsymbol{h} e^{j \phi[i]}\right) & =\rho M \frac{\rho}{\rho+1} \frac{2 \rho+1}{\rho+1} \\
& +\rho M\left(\frac{\rho}{\rho+1}\right)^{2} e^{-\sigma_{\theta}^{2} i}\left((M-1)-M e^{-\sigma_{\phi}^{2} i}\right) .
\end{aligned}
$$

In Figs. 4.1, 4.2 and 4.3 the achievable rates based on (4.27) and (4.30) are plotted for the sake of comparison. In all cases, the SI bound is better that the UNF bound. This is expected since the SI bound uses explicitly the estimated channel both for the data detection and data decoding. In contrast, the UNF bound in (4.30) uses the estimated channel knowledge only during data detection and "forgets" it during data decoding. In Fig. 4.1 the achievable rates at the first channel use, i.e., $i=1$, are plotted for $M=100$, $\sigma_{\phi}^{2}=\sigma_{\theta}^{2}=10^{-4}$ and $\tau_{d}=100$ as a function of $\rho,[\mathrm{dB}]$. The bounds are identical in the low $\rho$ regime, but the performance difference is substantial at high $\rho$. However, both bounds, SI and UNF, saturate at high $\rho$. In Fig. 4.2 the achievable rates (4.27) and (4.30) are plotted for $M=500$ and $i=100$. It is observed that the gap between the SI and UNF has decreased significantly. This is partly due to the larger $M$ and partly due the partial loss 
of the coherency between the estimated channel $\hat{\boldsymbol{h}}$ and the effective channel $\Theta[i] \boldsymbol{h} e^{j \phi[i]}$. At the 100-th data channel use the phase noise processes have evolved substantially so that omitting the information on $\hat{\boldsymbol{h}}$ during data decoding does not have a significant impact on the achievable rate performance. In Fig. 4.3 the average achievable rates over the coherence interval (calculated similarly to (4.22) using (4.27) and (4.30)) are plotted for $M=100, \tau_{d}=500$ and $\sigma_{\phi}^{2}=\sigma_{\theta}^{2}=10^{-3}$ and the bounds appear to be very close to each other.

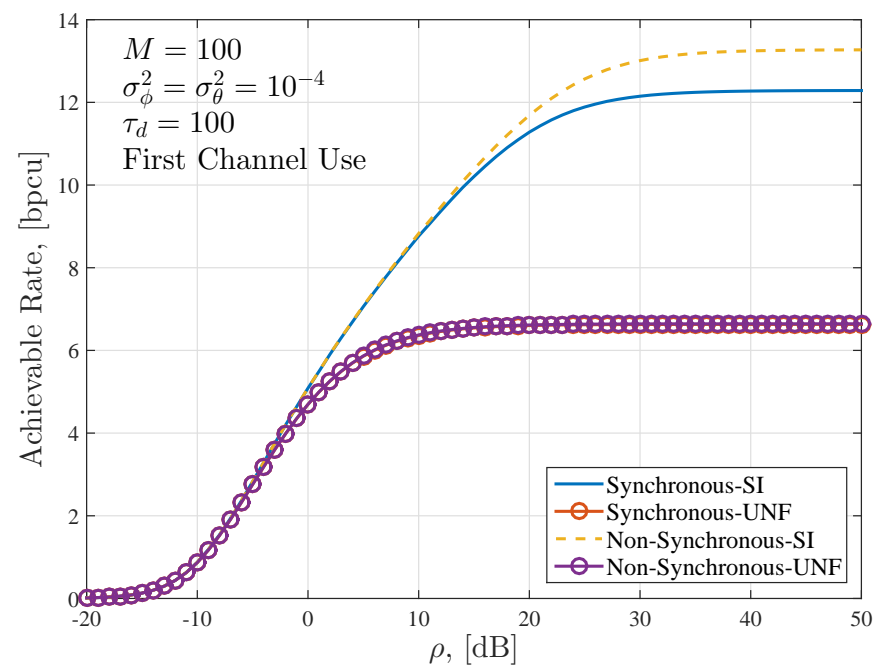

Figure 4.1: Comparison of the achievable rates in (4.27) and (4.30) for $i=1$, $M=100, \sigma_{\phi}^{2}=\sigma_{\theta}^{2}=10^{-4}$ and $\tau_{d}=100$ as a function of $\rho,[\mathrm{dB}]$.

In summary, in the examples it was observed that the SI bound yields better performance than the UNF bound. However, it also has several drawbacks. First, it is not in closed form and in particular for the non-synchronous operation the derivation of a closed-form expression appears to be formidable. Hence, we have to rely on Monte-Carlo simulations. The approach is difficult to be generalized for more practical scenarios, e.g., multi-user scenarios or longer training sequences. In particular, in the presence of multiple users the channel estimates are expected to be more noisy, hence the gain in achievable rate by exploiting the explicit form of the channel estimate, if this is possible, is likely to be very small. Also, the two bounds are far from each other only at high SNR, for small $M$, small phase noise variance, $\sigma_{\phi}^{2}, \sigma_{\theta}^{2}$, and for the initial channel uses of the coherence interval. For larger 


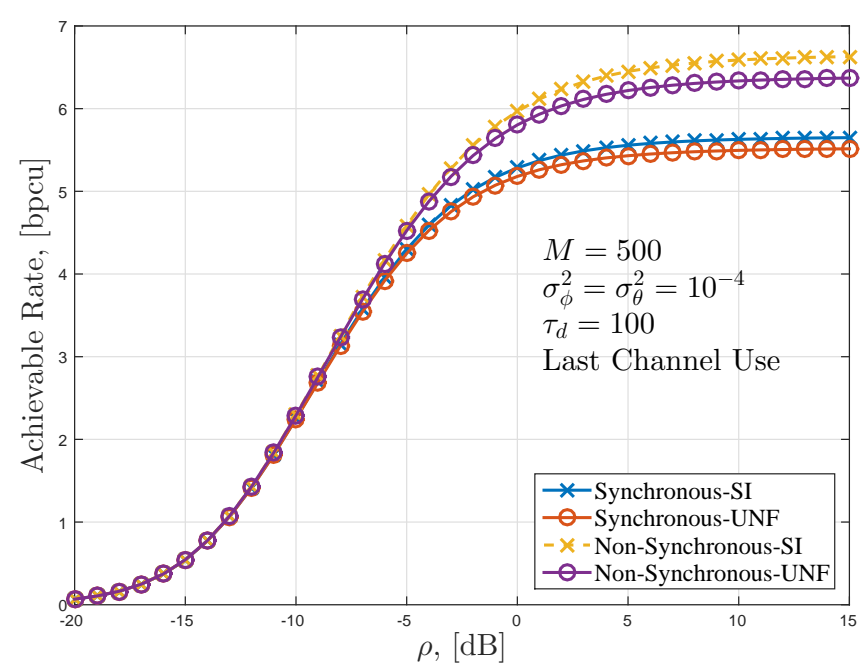

Figure 4.2: Comparison of the achievable rates in (4.27) and (4.30) for $i=$ $100, M=500, \sigma_{\phi}^{2}=\sigma_{\theta}^{2}=10^{-4}$ and $\tau_{d}=100$ as a function of $\rho,[\mathrm{dB}]$.

$M$, larger phase noise variances and longer coherence intervals, the performance difference between the SI bound and UNF bound is very small even in the scenario examined here.

The sum-capacity lower bounds presented in this dissertation were derived similarly to the approach outlined in Section 4.3. Based on Section 4.4 there is evidence that they are very close to the sum-capacity lower bound obtained if the estimated CSI was used not only for processing of the received signal but also for decoding of the information symbol. At the same time the bounds summarize concisely the influence of multiple parameters on the achievable rate performance of Massive MIMO with phase noise, without the need to resort to time-consuming Monte-Carlo simulations. 


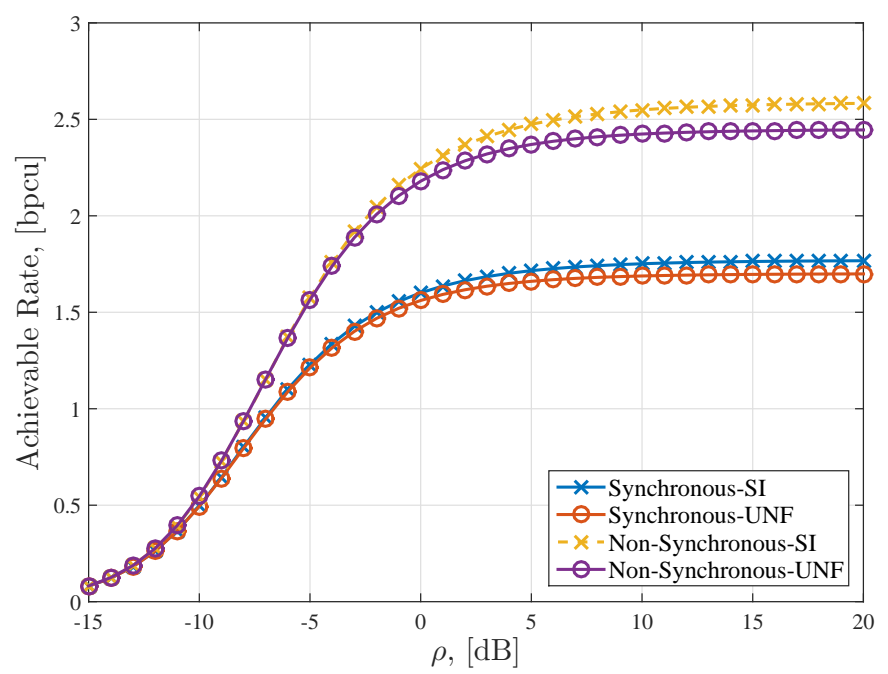

Figure 4.3: Comparison of the average achievable rates in (4.27) and (4.30) for $M=100, \sigma_{\phi}^{2}=\sigma_{\theta}^{2}=10^{-3}$ and $\tau_{d}=500$ as a function of $\rho,[\mathrm{dB}]$. 


\section{Chapter 5}

\section{Contributions of the Dissertation}

This dissertation focuses on the performance of Massive MIMO in frequency-selective channels and in the presence of phase noise impairments. The achievable rate performance of a frequency-selective Massive MIMO downlink is studied and compared with multi-carrier transmission. The achievable rate performance of a frequency-selective Massive MIMO uplink is studied in the presence of phase noise and estimated channel impulse responses is also analyzed. The study is extended to a frequency-flat phase-noise-impaired Massive MIMO uplink with zero-forcing receivers. The maximum-likelihood detector in a frequency-flat phase-noise-impaired SIMO channel is studied in the presence of training-based estimated channel impulse responses. The achievable rate performance of a phase-noiseimpaired Massive MIMO-OFDM uplink is also studied.

\subsection{Included Papers}

Brief summaries of the papers included in this dissertation are as follows:

Paper A: On the Optimality of Single-Carrier Transmission in LargeScale Antenna Systems 
Authored by Antonios Pitarokoilis, Saif Khan Mohammed, and Erik G. Larsson.

Published in the IEEE Wireless Communications Letters, 2012 [71].

A single carrier transmission scheme is presented for the frequency selective multi-user (MU) multiple-input single-output (MISO) Gaussian Broadcast Channel (GBC) with a base station (BS) having $M$ antennas and $K$ single antenna users. The proposed transmission scheme has low complexity and for $M \gg K$ it is shown to achieve near optimal sum-rate performance at low transmit power to receiver noise power ratio. Additionally, the proposed transmission scheme results in an equalization-free receiver and does not require any MU resource allocation and associated control signaling overhead. Also, the sum-rate achieved by the proposed transmission scheme is shown to be independent of the channel power delay profile (PDP). In terms of power efficiency, the proposed transmission scheme also exhibits an $O(M)$ array power gain. Simulations are used to confirm analytical observations.

\section{Paper B: Uplink Performance of Time-Reversal MRC in Massive MIMO Systems Subject to Phase Noise}

Authored by Antonios Pitarokoilis, Saif Khan Mohammed, and Erik G. Larsson.

Published in the IEEE Transactions on Wireless Communications, 2015 [72]. This work is an extension of the conference paper [73].

Multi-user multiple-input multiple-output (MU-MIMO) cellular systems with an excess of base station (BS) antennas (Massive MIMO) offer unprecedented multiplexing gains and radiated energy efficiency. Oscillator phase noise is introduced in the transmitter and receiver radio frequency chains and severely degrades the performance of communication systems. We study the effect of oscillator phase noise in frequency-selective Massive MIMO systems with imperfect channel state information (CSI). In particular, we consider two distinct operation modes, namely when the phase 
noise processes at the $M$ BS antennas are identical (synchronous operation) and when they are independent (non-synchronous operation). We analyze a linear and low-complexity time-reversal maximum-ratio combining (TR-MRC) reception strategy. For both operation modes we derive a lower bound on the sum-capacity and we compare their performance. Based on the derived achievable sum-rates, we show that with the proposed receive processing an $O(\sqrt{M})$ array gain is achievable. Due to the phase noise drift the estimated effective channel becomes progressively outdated. Therefore, phase noise effectively limits the length of the interval used for data transmission and the number of scheduled users. The derived achievable rates provide insights into the optimum choice of the data interval length and the number of scheduled users.

\section{Paper C: Achievable Rates of ZF Receivers in Massive MIMO with Phase Noise Impairments}

Authored by Antonios Pitarokoilis, Saif Khan Mohammed, and Erik G. Larsson.

Published in the proceedings of the Asilomar Conference on Signals, Systems and Computers, 2013 [74].

The effect of oscillator phase noise on the sum-rate performance of large multi-user multiple-input multiple-output (MU-MIMO) systems is studied. A Rayleigh fading MU-MIMO uplink channel is considered, where channel state information (CSI) is acquired via training. The base station (BS), which is equipped with an excess of antenna elements, $M$, uses the channel estimate to perform zero-forcing $(\mathrm{ZF})$ detection. A lower bound on the sum-rate performance is derived. It is shown that the proposed receiver structure exhibits an $O(\sqrt{M})$ array power gain. Additionally, the proposed receiver is compared with earlier studies that employ maximum ratio combining and it is shown that it can provide significant sum-rate performance gains at the medium and high signal-to-noise-ratio (SNR) regime. Further, the expression of the achievable sum rate provides new insights on the effect of various parameters on the overall system performance.

\section{Paper D: ML Detection in Phase Noise Impaired SIMO Channels with Uplink Training}


Authored by Antonios Pitarokoilis, Emil Björnson, and Erik G. Larsson.

Published in the IEEE Transactions on Communications, 2016 [75]. This is an extension of the conference paper [76].

The problem of maximum likelihood (ML) detection in training-assisted single-input multiple-output (SIMO) systems with phase noise impairments is studied for two different scenarios, i.e. the case when the channel is deterministic and known (constant channel) and the case when the channel is stochastic and unknown (fading channel). Further, two different operations with respect to the phase noise sources are considered, namely, the case of identical phase noise sources and the case of independent phase noise sources over the antennas. In all scenarios the optimal detector is derived for a very general parameterization of the phase noise distribution. Further, a high signal-to-noise-ratio (SNR) analysis is performed to show that symbol-error-rate (SER) floors appear in all cases. The SER floor in the case of identical phase noise sources (for both constant and fading channels) is independent of the number of antenna elements. In contrast, the SER floor in the case of independent phase noise sources is reduced when increasing the number of antenna elements (for both constant and fading channels). Finally, the system model is extended to multiple data channel uses and it is shown that the conclusions are valid for these setups, as well.

\section{Paper E: Performance of the Massive MIMO Uplink with OFDM and Phase Noise}

Authored by Antonios Pitarokoilis, Emil Björnson, and Erik G. Larsson.

Submitted to the IEEE Communications Letters.

The performance of multi-user Massive MIMO-OFDM uplink systems in the presence of base station (BS) phase noise impairments is investigated. Closed-form achievable rate expressions are rigorously derived under two different operations, namely the case of a common oscillator (synchronous operation) at the BS and the case of independent oscillators at each BS antenna (non-synchronous operation). It is observed that the nonsynchronous operation exhibits superior performance due to the averaging of intercarrier interference. Further, radiated power scaling laws are derived, which are identical to the phase-noise-free case. 


\subsection{Not Included Papers}

The following publications by the author are not included in the dissertation either because they do not fit within the main scope of the dissertation, or they were earlier versions of the journal publications included in the dissertation.

- A. Pitarokoilis, S. K. Mohammed, and E. G. Larsson, 'Effect of oscillator phase noise on uplink performance of large MU-MIMO systems', in Proc. 50th Annual Allerton Conference on Communication, Control, and Computing (Allerton), 2012, Monticello, IL, USA, pp. 1190-1197, Oct. 2012

- A. Pitarokoilis, E. Björnson, and E. G. Larsson, 'Optimal Detection in Training Assisted SIMO Systems with Phase Noise Impairments', in IEEE International Conference on Communications (ICC), London, U.K., pp. 2597-2602, June 2015

- E. Björnson, M. Matthaiou, A. Pitarokoilis, and E. G. Larsson, 'Distributed Massive MIMO in Cellular Networks: Impact of Imperfect Hardware \& Number of Oscillators', in 23rd European Signal Processing Conference (EUSIPCO), 2015, Nice, France, pp. 2436-2440, Aug. 31 2015-Sept. 42015

\subsection{Future Research Directions}

In this dissertation various aspects of Massive MIMO systems with wideband transmission and phase noise impairments are investigated. However, there are still many problems that are worthwhile to be investigated. In addition, the observations made here give rise to new research questions. In this section, we attempt to identify problems that are related with the present dissertation and could be considered by researchers in the future.

- In Paper A it was shown that single-carrier downlink transmission with perfect CSI at the BS can be close to optimal in the low spectral efficiency regime. The work could be extended to the imperfect CSI 
case and to the uplink transmission. In multi-cell frequency-flat Massive MIMO systems pilot contamination appears due to finite length of the coherence interval. It is reasonable to ask whether pilot contamination persists in frequency-selective channels. Can the frequency selectivity of the channel be used in order to suppress pilot contamination from users in neighboring cells?

- In Paper B and Paper C achievable sum-rates were derived for frequency-selective and frequency-flat uplink Massive MIMO systems with phase noise impairments. It is worthwhile to extend the results to the frequency-selective downlink and to other linear schemes, such as regularized zero forcing (RZF) precoding in the downlink or MMSE reception in the uplink. Towards this research direction, prior work includes [77], where the authors use asymptotic random matrix theory and provide capacity bounds for the case of frequency-flat downlink transmission with ZF precoding.

- In Paper B and Paper C the CSI was acquired via a simple training schedule, i.e., the training interval is at the beginning of the coherence interval and is not interleaved with the uplink data interval. Even though, the qualitative behavior of the achievable rates is not expected to change with some different scheduling of pilots, e.g., pilots at the end of the coherence interval or pilots interleaved with data transmission, it is useful to investigate whether there is an optimal pilot scheduling. Towards this direction, the authors in [69] have provided initial results for the flat-fading case and for more general modeling of hardware impairments that includes phase noise and additive hardware impairments.

- The derived achievable rates in Paper B and Paper C assumed that no phase noise estimation and compensation scheme was employed. However, it is reasonable to assume that there might be some phase noise tracking mechanism, such as the one proposed in [78]. The investigation of the gains in terms of achievable rate with phase noise compensation is a very important research topic. In [79] a simulationbased approach for the calculation of achievable rates is provided for a SISO AWGN phase noise channel. The extension of this work to Massive MIMO is particularly challenging and highly non-trivial, as the system model must be augmented with the effects of imperfect CSI, multi-user interference and channel fading.

- In Paper D the optimal, in the ML sense, detector with training based 
CSI was derived for a basic system model. It is important to investigate whether similar conclusions can be drawn in the downlink or if there is any fundamental difference. Also, in prior work, suboptimal algorithms based on factor graphs $[80,81]$ that perform well for single-user SISO [82] and MIMO [83] have been investigated. However, the provided algorithms appear to be too complex for Massive MIMO systems. Nevertheless, the works in [82], [83] and Paper D can be used as a starting point for novel, low complexity detection algorithms that are tailored to multi-user Massive MIMO setups with estimated CSI.

- In Paper E achievable rates were derived on Massive MIMO-OFDM uplink systems with phase noise impairments when perfect knowledge of the propagation channel is available at the receiver. The extension of the study to estimated CSI is a very relevant research problem. In the presence of phase noise the orthogonality of subchannels is destroyed and intercarrier interference appears. In addition, in OFDM systems often certain subcarriers in the same OFDM symbol are reserved for pilots and the rest for data transmission. Due to the presence of intercarrier interference, the estimated CSI is correlated with the transmitted data. This phenomenon complicates the analytical treatment of Massive MIMO with estimated CSI and phase noise.

- The Wiener phase noise model was used in the present dissertation. However, this model is an accurate description of free-running LOs with uncorrelated circuit noise sources. The analysis could be extended to more complex phase noise models that are tailored to LOs with certain spectral characteristics. Finally, recently the symbolspaced phase noise model is being revisited [33] and phase noise has been treated in the continuous time [84]. Also, in [34, 85, 86] multisample receivers for the Wiener phase noise channel have been studied and it was shown that an increase in the pre-log factor can be achieved. The extension of such receivers to the Massive MIMO setup could open new relevant research problems.

- The implementation of testbeds that could help verify the results presented in this dissertation is an important topic of study. Further, the outcome of these studies can help the researchers in communications to refine the applied models and make more accurate predictions of the true performance. 


\section{Bibliography}

[1] Cisco, "White paper: Cisco visual networking index: Global mobile data traffic forecast update, 2015-2020," tech. rep., February 2016.

[2] Ericsson, "White paper: 5G systems enabling industry and society transformation," Tech. Rep. Uen 284 23-3244, January 2015.

[3] E. Telatar, "Capacity of multi-antenna gaussian channels," European Transactions on Telecommunications, vol. 10, no. 6, pp. 585-595, 1999.

[4] G. Foschini and M. Gans, "On limits of wireless communications in a fading environment when using multiple antennas," Wireless Personal Communications, vol. 6, no. 3, pp. 311-335, Mar. 1998.

[5] D. Gesbert, M. Kountouris, R. W. Heath Jr., C.-B. Chae, and T. Sälzer, "Shifting the MIMO paradigm," IEEE Signal Processing Magazine, vol. 24, pp. 36-46, Sept. 2007.

[6] J. Winters, "Optimum combining for indoor radio systems with multiple users," IEEE Transactions on Communications, vol. 35, pp. 1222-1230, Nov. 1987.

[7] S. C. Swales, M. A. Beach, D. J. Edwards, and J. P. McGeehan, "The performance enhancement of multibeam adaptive base-station antennas for cellular land mobile radio systems," IEEE Transactions on Vehicular Technology, vol. 39, pp. 56-67, Feb. 1990.

[8] S. Anderson, M. Millnert, M. Viberg, and B. Wahlberg, "An adaptive array for mobile communication systems," IEEE Transactions on Vehicular Technology, vol. 40, pp. 230-236, Feb. 1991. 
[9] T. L. Marzetta, "Noncooperative cellular wireless with unlimited numbers of base station antennas," IEEE Transactions on Wireless Communications, vol. 9, pp. 3590-3600, Nov. 2010.

[10] H. Q. Ngo, E. G. Larsson, and T. L. Marzetta, "Energy and spectral efficiency of very large multiuser MIMO systems," IEEE Transactions on Communications, vol. 61, pp. 1436-1449, Apr. 2013.

[11] J. Hoydis, S. ten Brink, and M. Debbah, "Massive MIMO in the UL/DL of cellular networks: How many antennas do we need?," IEEE Journal on Selected Areas in Communications, vol. 31, pp. 160-171, Feb. 2013.

[12] G. Gonzalez, Foundations of Oscillator Circuit Design. Norwood, MA, USA: Artech House, Inc., 2006.

[13] A. Hajimiri and T. Lee, "A general theory of phase noise in electrical oscillators," IEEE Journal of Solid-State Circuits, vol. 33, pp. 179-194, Feb. 1998.

[14] A. Demir, A. Mehrotra, and J. Roychowdhury, "Phase noise in oscillators: a unifying theory and numerical methods for characterization," IEEE Transactions on Circuits and Systems I: Fundamental Theory and Applications, vol. 47, pp. 655-674, May 2000.

[15] J. G. Proakis, Digital Communications. McGraw-Hill, 1995.

[16] G. Kaplan and U. Ram, "Bounds on performance for the noisy reference PSK channel," IEEE Transactions on Communications, vol. 38, pp. 1699-1707, Oct. 1990.

[17] P. Y. Kam, S. Teo, Y. K. Some, and T. T. Tjhung, "Approximate results for the bit error probability of binary phase shift keying with noisy phase reference," IEEE Transactions on Communications, vol. 41, pp. 1020-1022, July 1993.

[18] B. Razavi, "A study of phase noise in CMOS oscillators," IEEE Journal of Solid-State Circuits, vol. 31, pp. 331-343, Mar. 1996.

[19] T. Lee, The Design of CMOS Radio-Frequency Integrated Circuits. Cambridge University Press, 2004.

[20] J. B. Johnson, "Thermal agitation of electricity in conductors," Physical Review, vol. 32, pp. 97-109, July 1928. 
[21] H. Nyquist, "Thermal agitation of electric charge in conductors," Physical Review, vol. 32, pp. 110-113, July 1928.

[22] W. Schottky, "Über spontane Stromschwankungen in verschiedenen Elektrizitätsleitern," Annalen der Physik, vol. 362, no. 23, pp. 541-567, 1918.

[23] M.S. Keshner, "1/f noise," Proceedings of the IEEE, vol. 70, pp. 212-218, Mar. 1982.

[24] B. Mandelbrot, "Some noises with $1 / f$ spectrum, a bridge between direct current and white noise," IEEE Transactions on Information Theory, vol. 13, pp. 289-298, Apr. 1967.

[25] D. Leeson, "A simple model of feedback oscillator noise spectrum," Proceedings of the IEEE, vol. 54, pp. 329-330, Feb. 1966.

[26] T. Lee and A. Hajimiri, "Oscillator phase noise: a tutorial," IEEE Journal of Solid-State Circuits, vol. 35, pp. 326-336, Mar. 2000.

[27] J. H. Dauwels, On graphical models for communications and machine learning: algorithms, bounds, and analog implementation. PhD thesis, ETH Zürich, Hartung-Gorre Verlag, Konstanz, 2006. Diss., Eidgenössische Technische Hochschule ETH Zürich, Nr. 16365, 2005.

[28] D. Petrovic, W. Rave, and G. Fettweis, "Effects of phase noise on OFDM systems with and without PLL: Characterization and compensation," IEEE Transactions on Communications, vol. 55, pp. 1607 -1616, Aug. 2007.

[29] P. Billingsley, Probability and Measure, Anniversary Edition. Wiley, 2012.

[30] A. Demir, "Phase noise in oscillators: DAEs and colored noise sources," in IEEE/ACM International Conference on Computer-Aided Design, 1998. ICCAD 98. Digest of Technical Papers. 1998, pp. 170-177, Nov. 1998.

[31] H. L. van Trees, Detection, Estimation, and Modulation Theory, Part I. New York: Wiley Interscience, 2001.

[32] G. Colavolpe, "Communications over phase-noise channels: A tutorial review," in 6th Advanced Satellite Multimedia Systems Conference (ASMS) and 12th Signal Processing for Space Communications Workshop (SPSC), 2012, pp. 316-327, Sept. 2012. 
[33] M. Martalò, C. Tripodi, and R. Raheli, "On the information rate of phase noise-limited communications," in Information Theory and Applications Workshop (ITA), 2013, pp. 1-7, Feb. 2013.

[34] H. Ghozlan and G. Kramer, "Models and information rates for Wiener phase noise channels," CoRR, vol. abs/1503.03130, 2015.

[35] T. Pollet, M. Van Bladel, and M. Moeneclaey, "BER sensitivity of OFDM systems to carrier frequency offset and wiener phase noise," IEEE Transactions on Communications, vol. 43, pp. 191-193, Feb/Mar/Apr 1995.

[36] L. Tomba, "On the effect of Wiener phase noise in OFDM systems," IEEE Transactions on Communications, vol. 46, pp. 580-583, May 1998.

[37] Q. Zou, A. Tarighat, and A. Sayed, "Compensation of phase noise in OFDM wireless systems," IEEE Transactions on Signal Processing, vol. 55, pp. 5407-5424, Nov. 2007.

[38] T. Schenk, RF Imperfections in High-rate Wireless Systems: Impact and Digital Compensation. Springer, 2008.

[39] P. Mathecken, T. Riihonen, S. Werner, and R. Wichman, "Performance analysis of OFDM with wiener phase noise and frequency selective fading channel," IEEE Transactions on Communications, vol. 59, pp. 1321-1331, May 2011.

[40] P. Mathecken, T. Riihonen, N. Tchamov, S. Werner, M. Valkama, and R. Wichman, "Characterization of OFDM radio link under PLL-based oscillator phase noise and multipath fading channel," IEEE Transactions on Communications, vol. 60, pp. 1479-1485, June 2012.

[41] A. Viterbi, Principles of Coherent Communication. McGraw-Hill Inc., 1966.

[42] G. J. Foschini, R. D. Gitlin, and S. B. Weinstein, "On the selection of a two-dimensional signal constellation in the presence of phase jitter and Gaussian noise," Bell System Technical Journal, vol. 52, pp. 927-965, July-Aug. 1973.

[43] A. Lapidoth, "On phase noise channels at high SNR," in Proc. of the 2002 IEEE Information Theory Workshop, pp. 1-4, Oct. 2002. 
[44] B. Goebel, R.-J. Essiambre, G. Kramer, P. J. Winzer, and N. Hanik, “Calculation of mutual information for partially coherent Gaussian channels with applications to fiber optics," IEEE Transactions on Information Theory, vol. 57, pp. 5720-5736, Sept. 2011.

[45] G. Durisi, A. Tarable, C. Camarda, R. Devassy, and G. Montorsi, “Capacity bounds for MIMO microwave backhaul links affected by phase noise," IEEE Transactions on Communications, vol. 62, pp. 920-929, Mar. 2014.

[46] D. Tse and P. Viswanath, Fundamentals of Wireless Communication. Cambridge, UK: Cambridge Univ. Press, 2004.

[47] A. E. Gamal and Y.-H. Kim, Network Information Theory. New York, NY, USA: Cambridge University Press, 2012.

[48] A. Goldsmith, S. A. Jafar, N. Jindal, and S. Vishwanath, "Capacity limits of MIMO channels," IEEE Journal on Selected Areas in Communications, vol. 21, pp. 684-702, June 2003.

[49] P. Viswanath, D. N. C. Tse, and V. Anantharam, "Asymptotically optimal water-filling in vector multiple-access channels," IEEE Transactions on Information Theory, vol. 47, pp. 241-267, Jan 2001.

[50] L. Li and A. J. Goldsmith, "Capacity and optimal resource allocation for fading broadcast channels .I. ergodic capacity," IEEE Transactions on Information Theory, vol. 47, pp. 1083-1102, Mar. 2001.

[51] L. Li and A. J. Goldsmith, "Capacity and optimal resource allocation for fading broadcast channels .II. outage capacity," IEEE Transactions on Information Theory, vol. 47, pp. 1103-1127, Mar. 2001.

[52] G. Caire and S. Shamai, "On the achievable throughput of a multiantenna gaussian broadcast channel," IEEE Transactions on Information Theory, vol. 49, pp. 1691-1706, July 2003.

[53] P. Viswanath and D. N. C. Tse, "Sum capacity of the vector gaussian broadcast channel and uplink-downlink duality," IEEE Transactions on Information Theory, vol. 49, pp. 1912-1921, Aug. 2003.

[54] S. Vishwanath, N. Jindal, and A. Goldsmith, "Duality, achievable rates, and sum-rate capacity of gaussian MIMO broadcast channels," IEEE Transactions on Information Theory, vol. 49, pp. 2658-2668, Oct. 2003. 
[55] W. Yu and J. M. Cioffi, "Sum capacity of gaussian vector broadcast channels," IEEE Transactions on Information Theory, vol. 50, pp. 18751892, Sept. 2004.

[56] N. Jindal, S. Vishwanath, and A. Goldsmith, "On the duality of gaussian multiple-access and broadcast channels," IEEE Transactions on Information Theory, vol. 50, pp. 768-783, May 2004.

[57] H. Weingarten, Y. Steinberg, and S. Shamai, "The capacity region of the gaussian multiple-input multiple-output broadcast channel," IEEE Transactions on Information Theory, vol. 52, pp. 3936-3964, Sept. 2006.

[58] F. Rusek, D. Persson, B. K. Lau, E. G. Larsson, T. L. Marzetta, O. Edfors, and F. Tufvesson, "Scaling up MIMO: Opportunities and challenges with very large arrays," IEEE Signal Processing Magazine, vol. 30, pp. 40-60, Jan. 2013.

[59] E. G. Larsson, F. Tufvesson, O. Edfors, and T. L. Marzetta, "Massive MIMO for next generation wireless systems," IEEE Communications Magazine, vol. 52, pp. 186-195, Feb. 2014.

[60] H. Q. Ngo, E. G. Larsson, and T. L. Marzetta, "Massive MU-MIMO downlink TDD systems with linear precoding and downlink pilots," in Communication, Control, and Computing (Allerton), 2013 51st Annual Allerton Conference on, pp. 293-298, Oct 2013.

[61] H. Q. Ngo, Massive MIMO: Fundamentals and System Designs. Linköping Studies in Science and Technology. Dissertations No. 1642, Linköping University, Sweden, 2015.

[62] M. Médard, "The effect upon channel capacity in wireless communications of perfect and imperfect knowledge of the channel," IEEE Transactions on Information Theory, vol. 46, pp. 933-946, May 2000.

[63] A. Lapidoth and S. Shamai, "Fading channels: how perfect need "perfect side information" be?," IEEE Transactions on Information Theory, vol. 48, pp. 1118-1134, May 2002.

[64] B. Hassibi and B. Hochwald, "How much training is needed in multiple-antenna wireless links?," IEEE Transactions on Information Theory, vol. 49, pp. 951 - 963, Apr. 2003.

[65] T. L. Marzetta, "How much training is required for multiuser MIMO?," in Proc. 40th Asilomar Conference on Signals, Systems and Computers, pp. $359-363$, Nov. 2006. 
[66] J. Jose, A. Ashikhmin, T. L. Marzetta, and S. Vishwanath, "Pilot contamination and precoding in multi-cell TDD systems," IEEE Transactions on Wireless Communications, vol. 10, pp. 2640-2651, Aug. 2011.

[67] E. Björnson, J. Hoydis, M. Kountouris, and M. Debbah, "Massive MIMO systems with non-ideal hardware: Energy efficiency, estimation, and capacity limits," IEEE Transactions on Information Theory, vol. 60, pp. 7112-7139, Nov. 2014.

[68] H. Yang and T. L. Marzetta, "Performance of conjugate and zeroforcing beamforming in large-scale antenna systems," IEEE Journal on Selected Areas in Communications, vol. 31, pp. 172-179, Feb. 2013.

[69] E. Björnson, M. Matthaiou, and M. Debbah, “Massive MIMO with nonideal arbitrary arrays: Hardware scaling laws and circuit-aware design," IEEE Transactions on Wireless Communications, vol. 14, pp. 43534368, Aug. 2015.

[70] E. Björnson, M. Matthaiou, A. Pitarokoilis, and E. G. Larsson, "Distributed Massive MIMO in cellular networks: Impact of imperfect hardware and number of oscillators," in 23rd European Signal Processing Conference (EUSIPCO), 2015, pp. 2436-2440, Aug. 2015.

[71] A. Pitarokoilis, S. K. Mohammed, and E. G. Larsson, "On the optimality of single-carrier transmission in large-scale antenna systems," IEEE Wireless Communications Letters, vol. 1, pp. 276 -279, Aug. 2012.

[72] A. Pitarokoilis, S. K. Mohammed, and E. G. Larsson, "Uplink performance of time-reversal MRC in massive MIMO systems subject to phase noise," IEEE Transactions on Wireless Communications, vol. 14, pp. 711-723, Feb. 2015.

[73] A. Pitarokoilis, S. K. Mohammed, and E. G. Larsson, "Effect of oscillator phase noise on the uplink performance of large MU-MIMO systems," in Proc. of the 50th Allerton Conference on Communication Control and Computing, pp. 1190-1197, Oct. 2012.

[74] A. Pitarokoilis, S. K. Mohammed, and E. G. Larsson, "Achievable rates of ZF receivers in massive MIMO with phase noise impairments," in Proc. of the 47th Asilomar Conference on Signals, Systems and Computers, 2013, pp. 1004-1008, Nov. 2013. 
[75] A. Pitarokoilis, E. Björnson, and E. G. Larsson, "ML detection in phase noise impaired SIMO channels with uplink training," IEEE Transactions on Communications, vol. 64, pp. 223-235, Jan. 2016.

[76] A. Pitarokoilis, E. Björnson, and E. G. Larsson, "Optimal detection in training assisted SIMO systems with phase noise impairments," in IEEE International Conference on Communications (ICC), 2015, pp. 25972602, June 2015.

[77] R. Krishnan, M. Khanzadi, N. Krishnan, Y. Wu, A. Graell i Amat, T. Eriksson, and R. Schober, "Linear Massive MIMO precoders in the presence of phase noise - a large-scale analysis," IEEE Transactions on Vehicular Technology, vol. PP, no. 99, pp. 1-1, 2015.

[78] H. Mehrpouyan, A. A. Nasir, S. D. Blostein, T. Eriksson, G. K. Karagiannidis, and T. Svensson, "Joint estimation of channel and oscillator phase noise in MIMO systems," IEEE Transactions on Signal Processing, vol. 60, pp. 4790-4807, Sept. 2012.

[79] L. Barletta, M. Magarini, S. Pecorino, and A. Spalvieri, "Upper and lower bounds to the information rate transferred through first-order markov channels with free-running continuous state," IEEE Transactions on Information Theory, vol. 60, pp. 3834-3844, July 2014.

[80] F. R. Kschischang, B. J. Frey, and H. A. Loeliger, "Factor graphs and the sum-product algorithm," IEEE Transactions on Information Theory, vol. 47, pp. 498-519, Feb 2001.

[81] H. A. Loeliger, "An introduction to factor graphs," IEEE Signal Processing Magazine, vol. 21, pp. 28-41, Jan 2004.

[82] G. Colavolpe, A. Barbieri, and G. Caire, "Algorithms for iterative decoding in the presence of strong phase noise," IEEE Journal on Selected Areas in Communications, vol. 23, pp. 1748-1757, Sept. 2005.

[83] R. Krishnan, G. Colavolpe, A. Graell i Amat, and T. Eriksson, "Algorithms for joint phase estimation and decoding for MIMO systems in the presence of phase noise and quasi-static fading channels," IEEE Transactions on Signal Processing, vol. 63, pp. 3360-3375, July 2015.

[84] L. Barletta and G. Kramer, "On continuous-time white phase noise channels," in 2014 IEEE International Symposium on Information Theory, pp. 2426-2429, June 2014. 
[85] H. Ghozlan and G. Kramer, "Multi-sample receivers increase information rates for Wiener phase noise channels," in 2013 IEEE Global Communications Conference (GLOBECOM), pp. 1897-1902, Dec 2013.

[86] H. Ghozlan and G. Kramer, "Phase modulation for discrete-time Wiener phase noise channels with oversampling at high SNR," in 2014 IEEE International Symposium on Information Theory, pp. 1554-1557, June 2014. 


\section{Part II}

\section{Included Papers}





\section{Included Papers}

The articles associated with this thesis have been removed for copyright reasons. For more details about these see:

http://urn.kb.se/resolve?urn=urn:nbn:se:liu:diva-127399 



\section{Linköping Studies in Science and Technology Dissertations, Division of Communication Systems Department of Electrical Engineering (ISY) Linköping University, Sweden}

Erik Axell, Spectrum Sensing Algorithms Based on Second-Order Statistics, Dissertation No. 1457, 2012.

Tumula V. K. Chaitanya, HARQ Systems: Resource Allocation, Feedback Error Protection, and Bits-to-Symbol Mappings, Dissertation No. 1526, 2013.

Johannes Lindblom, The MISO Interference Channel as a Model for Non-Orthogonal Spectrum Sharing, Dissertation No. 1555, 2013.

Reza Moosavi, Improving the Efficiency of Control Signaling in Wireless Multiple Access Systems, Dissertation No. 1556, 2014.

Mirsad Čirkić, Efficient MIMO Detection Methods, Dissertation No. 1570, 2014.

Hien Quoc Ngo, Massive MIMO: Fundamentals and System Designs, Dissertation No. $1642,2015$.

Anu Kalidas Muralidharan Pillai, Signal Reconstruction Algorithms for TimeInterleaved ADCs, Dissertation No. 1672, 2015. 